\title{
WestVirginiaUniversity
}

THE RESEARCH REPOSITORY @ WVU

Graduate Theses, Dissertations, and Problem Reports

2011

\section{Traffic Optimization for Multimodal Cooperative Networks}

Krishna Jyothi Boppana

West Virginia University

Follow this and additional works at: https://researchrepository.wvu.edu/etd

\section{Recommended Citation}

Boppana, Krishna Jyothi, "Traffic Optimization for Multimodal Cooperative Networks" (2011). Graduate Theses, Dissertations, and Problem Reports. 4697.

https://researchrepository.wvu.edu/etd/4697

This Thesis is protected by copyright and/or related rights. It has been brought to you by the The Research Repository @ WVU with permission from the rights-holder(s). You are free to use this Thesis in any way that is permitted by the copyright and related rights legislation that applies to your use. For other uses you must obtain permission from the rights-holder(s) directly, unless additional rights are indicated by a Creative Commons license in the record and/ or on the work itself. This Thesis has been accepted for inclusion in WVU Graduate Theses, Dissertations, and Problem Reports collection by an authorized administrator of The Research Repository @ WVU. For more information, please contact researchrepository@mail.wvu.edu. 


\title{
Traffic Optimization for Multimodal Cooperative Networks
}

\author{
by \\ Krishna Jyothi Boppana \\ Thesis submitted to the \\ College of Engineering and Mineral Resources \\ at West Virginia University \\ in partial fulfillment of the requirements \\ for the degree of \\ Master of Science \\ in \\ Electrical Engineering \\ Matthew C. Valenti \\ Vinod K. Kulathumani \\ Daryl Reynolds, Ph.D., Chair
}

Lane Department of Computer Science and Electrical Engineering

Morgantown, West Virginia

2011

Keywords: Utility Function; Cooperative Diversity; Multimodal Network

Copyright 2011 Krishna Jyothi Boppana 


\begin{abstract}
Traffic Optimization for Multimodal Cooperative Networks

by
\end{abstract}

Krishna Jyothi Boppana

Resource allocation and traffic optimization are crucial problems in multi-traffic wireless networks as resources are scarce and the traffic is shared by multiple users. When application performance is the key concern in a network, utility is considered as a reliable metric. A lot of research has been done on capacity limits of wireless networks under some assumptions on the physics of propagation and some restrictions on the communication strategy employed by the nodes. It has been proved that due to the physical limitation that holds for any any communication system, the decrease in throughput problem as wireless nodes increase in a fixed area cannot be overcome. In this thesis, multimodal networks, where other modes of communication like wires, infrared links may operate in addition to wireless nodes to improve the performance of the wireless network are studied and rate and power optimization problems are considered.

In this thesis, rate and power optimizations for heterogeneous traffic multimodal networks are proposed by solving them separately. First, the total rate is divided between delay-sensitive data and voice to achieve maximum utility in the network and in the next step, we divide transmit power between the wireless channel and the wired channel for a source-destination pair multimodal network and a diamond cooperative multimodal network implemented with Lanemann protocol. We consider a utility function for delay-sensitive data as a function of rate, reliability and delay and, the utility function of voice is a function of rate and delay. Traffic optimization problem is then solved by maximizing the sum of utilities of all voice and data users in the network and optimal rates that can be allocated to data and voice are calculated. Power allocation for a source-destination pair multimodal networks is done by optimizing instantaneous rate and outage probability in the network. For implementing a cooperative wireless relay network, we consider two cases: wire place between source and relay and wire placed between relay and destination. Optimal power allocation to wired and wireless channels is done by analytically minimizing the high-SNR outage probability expressions. 
To my family 


\section{Acknowledgements}

I would like to take this opportunity to thank all the individuals who has supported me for the successful completion of my thesis. First of all, I am indebted to my advisor Dr. Daryl Reynolds for inspiration and timely advice throughout my thesis. I greatly appreciate his patience for answering numerous questions and helping me to tune my writing skills. I would like to thank Dr. Matthew Valenti and Dr. Vinod Kulathumani for fruitful discussions and for being on my graduate committee.

I am grateful to Dr. Reynolds and Dr. Mary Carter for giving me an opportunity to work as graduate assistant. I am thankful to my colleagues Ricky Hussmann and Harish Kumar Palakurthi for many helpful discussions.

I would like to thank my mother Swarupa, father Janardhana Rao, brother Krishna Chaitanya, and my sister-in-law Mounika for their love and constant support throughout my studies and career. I am thankful to all my roommates and my friends Krishna, Raghu, Spoorthi, Mohita, Srikanth, Jagadish, Savan, Kartheek, Eswar for making my graduate experience memorable. I am also thankful to my relatives and friends in United States and India for their love and encouragement. 


\section{Contents}

Acknowledgements $\quad$ iv

List of Figures $\quad$ vii

List of Tables $\quad$ viii

$\begin{array}{ll}\text { Notation } & \text { ix }\end{array}$

1 Introduction $\quad 1$

1.1 Thesis Outline . . . . . . . . . . . . . . . . . . . 3

2 Basic Concepts and Background $\quad 6$

2.1 Wireless Communication Concepts . . . . . . . . . . . . . . . . 6

2.1.1 Transmitted and Received Signal Models . . . . . . . . . . . . . 6

2.1.2 Wireless Channel . . . . . . . . . . . . . . . . . . 7

2.1.3 Small Scale Fading and Multipath . . . . . . . . . . . . . . 8

2.1.4 Rayleigh Fading Distribution . . . . . . . . . . . . . . . . 8

2.1.5 Maximum Ratio Combining . . . . . . . . . . . . . . . . . . . . . . 9

2.1.6 Space Time Coding . . . . . . . . . . . . . . . . . . . . 10

2.2 Capacity and Outage Probability . . . . . . . . . . . . . . . 11

2.2.1 Channel Capacity . . . . . . . . . . . . . . . . . . . 11

2.2.2 Capacity in an Additive White Gaussian Noise Channel . . . . . . . . 11

2.2.3 Capacity for Fading Channels . . . . . . . . . . . . . . . . . . 12

2.2.4 Information Outage Probability for Non-ergodic Channel . . . . . . . 13

2.3 Resource Allocation for Data and Voice . . . . . . . . . . . . . . . . . . . 14

2.3.1 Utility Function . . . . . . . . . . . . . . . . . . . 15

2.4 Cooperative Communications _ . . . . . . . . . . . . . . . 16

2.4.1 Cooperative Diversity Protocols . . . . . . . . . . . . . . . 18

2.4.2 Laneman's Repetition-Based Cooperative Diversity Protocol . . . . . 19

2.4.3 Laneman's Space-Time Coded Cooperative Diversity Protocol . . . . 20

2.5 Multimodal Networks . . . . . . . . . . . . . . . . . . . . . 20

2.6 Summary . . . . . . . . . . . . . . . . . . . . 23 
3 Traffic optimization in Heterogeneous Traffic Networks 25

3.1 Network Model . . . . . . . . . . . . . . . . . . . 26

3.1 .1 Utility Function for Data . . . . . . . . . . . . . 26

3.1 .2 Utility Function for Voice . . . . . . . . . . . . . . . . . . 27

3.2 Maximizing Total Utility using Lagrange Multipliers Method . . . . . . . . . 28

3.3 Numerical Results . . . . . . . . . . . . . . . . . . . . . . . . . . . . . . 29

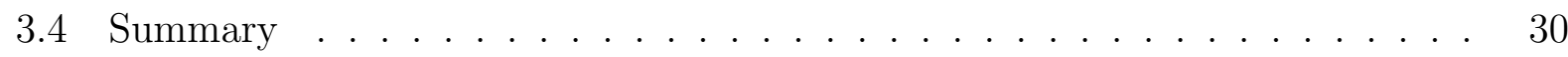

4 Power Allocation in a Point to Point Wireless Network with an Additional Mode

4.1 Network Model . . . . . . . . . . . . . . . . . . . . . . . . 32

4.2 Instantaneous Rate Maximization . . . . . . . . . . . . . . . . . 33

4.3 Outage Probability Minimization . . . . . . . . . . . . . . . . . . . . . . . . . . . . . 34

4.4 Numerical Results . . . . . . . . . . . . . . . . . . . . 35

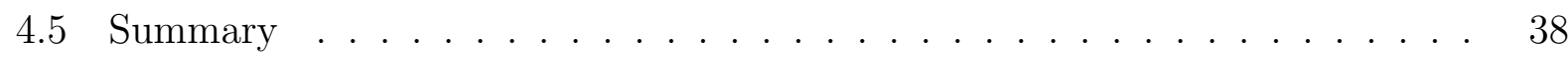

5 Outage Probability Minimization in a Cooperative Multimodal Network 40

5.1 Network Model . . . . . . . . . . . . . . . . . . . . . . . 41

5.2 Outage Probability for the Network with Wire between Source and Relay . . 42

5.3 Outage Probability for the Network with Wire between Relay and Destination 44

5.4 Numerical Results . . . . . . . . . . . . . . . . . . . . . . 45

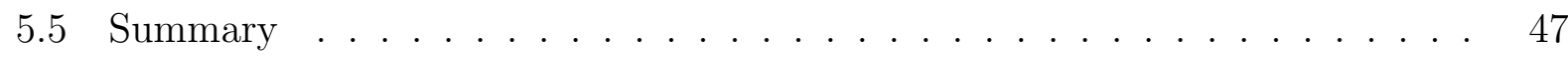

6 Conclusion and Future Work $\quad 48$

6.1 Summary and Conclusions . . . . . . . . . . . . . . . . 48

6.2 Future Work . . . . . . . . . . . . . . . . . . . . . . 49

References $\quad \mathbf{5 0}$

A Proof for Maximizing Total Utility in Data and Voice Networks . . . . . . . 54

B Proof for Rate Maximization in a Source-Destination Pair Multimodal Network 55

C Proof for Outage Minimization in a Source-Destination Pair Multimodal Net-

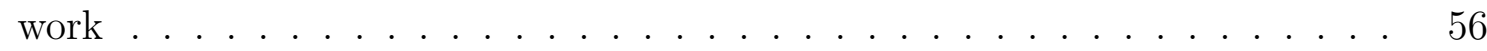

D Proofs for Minimizing Outage probability for Cooperative Diamond Relay Multimodal Network . . . . . . . . . . . . . . . . . . . 57

D.1 Wire between Source and Relay _. . . . . . . . . . . . . 57

D.2 Wire between Relay and Destination . . . . . . . . . . . . 58 


\section{List of Figures}

1.1 Block Diagram $1 \ldots \ldots \ldots \ldots \ldots$

1.2 Block Diagram $2 \ldots \ldots \ldots \ldots$. . . . . . . . . . . . . . . . 4

2.1 Capacity plot for a AWGN channel with increase in SNR . . . . . . . . . . . 12

2.2 Information outage probability plot for a AWGN channel with increase in SNR 14

2.3 Multimodal Network . . . . . . . . . . . . . . . . . . . . . . . . . . 21

2.4 Outage probability curves for diamond relay network with wire between source and relay for three different values of SNR . . . . . . . . . . . . 23

2.5 Outage probability curves for diamond relay network with wire between relay and destination for three different values of SNR . . . . . . . . . . . . . 24

3.1 Total utility plot for a network with increase in rate allocated to data traffic 30

4.1 Wireless network with additional mode of communication (wire) . . . . . . . 32

4.2 Plot of wired power vs total instantaneous rate for a multimodal network for various bandwidth ratios of wired and wireless channel . . . . . . . . . . 36

4.3 Plot of wired power vs total instantaneous rate for a multimodal network for different values of wireless gain $h \ldots \ldots . \ldots . \ldots 36$

4.4 Plot of wired power vs outage probability for a multimodal network for $B_{w}=$

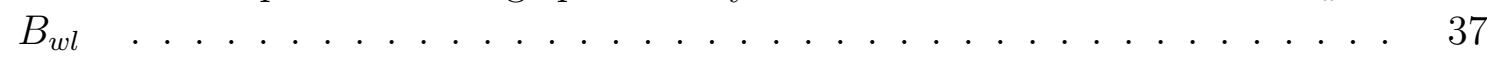

4.5 Plot of wired power vs outage probability for multimodal network for $B_{w}=$ $\frac{1}{10} B_{w l} \ldots \ldots \ldots \ldots \ldots \ldots$

4.6 Plot of wired power vs outage probability for multimodal network for $B_{w}=$

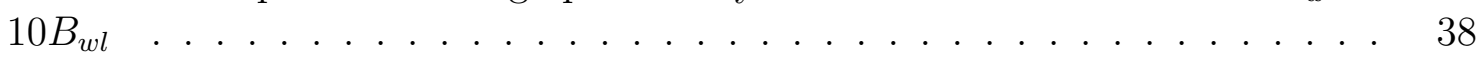

5.1 Cooperative relay network with wire between source and relay . . . . . . . . 41

5.2 Cooperative relay network with wire between relay and destination . . . . . 42

5.3 Outage probability plot for wire between source and relay for a diamond relay network with three power values, $12 \mathrm{~dB}$ (blue), 18dB (green), 24dB (red) and the points for the corresponding plots are the optimal power points . . . . .

5.4 Outage probability plot for wire between relay and destination for a diamond relay network with three power values, $12 \mathrm{~dB}$ (blue), 18dB (green), $24 \mathrm{~dB}$ (red) and the points for the corresponding plots are the optimal power points . 
List of Tables 


\section{Notation}

We use the following notation and symbols throughout this thesis.

$U(\cdot) \quad$ : Utility Function

$x * y \quad$ : Convolution between $x$ and $y$

$\Re\{\cdot\}$ : Real part of the argument

$|\cdot|$ : Cardinality of a set

$\hat{x} \quad$ : Estimates signal of $x$

$(\cdot)^{*} \quad$ : Complex conjugate

$\Im\{\cdot\}$ : Imaginary part of the argument

Bold uppercase letters denote matrices while bold lower case letters denote vectors. 


\section{Chapter 1}

\section{Introduction}

It is challenging to design a network which accommodates diverse applications and different kinds of traffic. For example, an IMT-advanced cellular system must have target peak data rates of up to approximately $100 \mathrm{Mbit} / \mathrm{s}$ for high mobility such as mobile access and up to approximately $1 \mathrm{Gbit} / \mathrm{s}$ for low mobility such as nomadic/local wireless access, according to the ITU requirements [1]. High data rates are mainly due to recent innovations in signal processing, modulation techniques and smart antennas that can directly transmit signal to users. But mobile users within the cell experience attenuation due to fading. Fading is variation of attenuation that a telecommunication signal experiences over a channel. Multipath propagation and shadowing are the causes for fading. Fading due to multipath is referred as multipath induced fading and fading due to shadowing from obstacles is referred as shadow fading. Fading decreases the data rates and SNR of the signal. Fading also results to the retransmission of signal which consumes more energy. In order to achieve high SNR at the receiving end fading should be mitigated using some methods.

Cooperative diversity techniques increase the SNR of the signal which in turn increases the performance of the system. In cooperative communications, users increase the effective quality of service by the cooperation between users. There are different types of cooperative diversity techniques for message transmission. Cooperation leads to the exciting tradeoffs in received SNR of the signal.

VoIP networks are introduced to run both data and voice communication in a single network. In recent years, a lot of research is being focused on the resource allocation problem 
in multi-traffic communication networks. The distribution of available rate to data packets in a network is a difficult problem as rate, reliability and delay requirements vary for different kinds of traffic. Voice and video are sensitive to delay while a delay-tolerant data service needs sufficient throughput. Traffic can be placed in a queue and rate may be allocated based on first come first serve basis. This is not a viable solution in some cases where some delay sensitive traffic may be held in queue while low priority data packets are being transmitted which increases delay thus decreasing efficiency of the system. Priority queuing [2] is one sophisticated scheme for allocating resources to the traffic with different QoS requirements. It puts all the packets together and allocates high priority to the packets that require less delay and transmits them according to their priority. In [3], a new scheme, batch and priority based admission control is proposed for multi-rate wireless systems to allocate bandwidth according to various QoS requirements of the traffic. Voice and data can be integrated using some fixed or adaptive boundaries that allocate data and voice in slots based on traffic requirement in integrated voice and data wireless networks $[4,5]$. Efficiency of resource allocation can be measured by the achieved network utility or fairness. Power and rate for wireless systems are allocated by solving a constrained optimization problem to achieve minimum transmit power and maximum rate [6]. In [7], network resource optimization in a relay-assisted cellular networks with heterogeneous QoS requirements is done by deriving two efficient algorithms. Zhou et al. $[8,9,10]$ proposed a resource allocation scheme called revenue maximization to maximize total profit in a multi-cell wireless multimedia network by selling resources to the users. For rate allocation, Network Utility Maximization (NUM) [11] can consider utility functions of all kinds of traffic and allocates rate to achieve their maximum combined utility. The NUM framework [12] has diverse applications in network rate allocating algorithms, in Internet congestion control protocols and network-fairness characterization. In [13], the best use of rate-reliability characteristics at the physical layer to support different kinds of traffic over a network and maximizing their utilities are studied. Stidham et al. [14] and La et al. [15] introduced adaptive rate-control algorithms for heterogeneous traffic users where users can adjust their rate flow based on utility function and delay sensitivity. In [16, 17], fairness is achieved in mobile multimedia DS-CDMA systems by allocating minimal transmission data rate to each user. Resource allocation in wireless LANs differ from cellular networks 
due to diversity in physical, medium access and link control layers. For use in 802.11 wireless networks, Visser et al. [18] proposed a protocol that sends data in a contention based access period and voice during a contention free period. In cellular/WLAN interworking, voice can be sent through cellular network and data can be sent through WLANs based on the characteristics of cellular network and WLANs and QoS requirements of voice and data traffic [19].

In [20], classical relay channel models are studied and capacities of gaussian relay channels and certain discrete relay channels are evaluated. In [21, 22], cooperative relay networks are developed and protocols required for transmitting and receiving signal are designed. In [23], it is shown that per-node throughput drops to zero as network size increases in a pure wireless network. Recent works show that the decrease in throughput is due to a physical limitation that holds for any communication system [24]. In [25], it is shown that the performance of a cooperative wireless relay network increases if we add a non-fading channel (wire), another mode of communication between the nodes, assuming some characteristics of wire. In this thesis, we consider multimodal networks, defined in [25] where wired and/or wireless modes may operate simultaneously and improve the performance of a wireless network.

\subsection{Thesis Outline}

In this thesis, we concentrate on resource and power allocation problems for heterogeneous traffic multimodal networks. Two types of traffic, delay-sensitive data and voice are considered and rate allocation is done maximizing their combined utility in the network. Power allocation is done for a source-destination pair multimodal network to achieve maximum capacity and minimum outage probability separately. For a cooperative diamond relay multimodal network, high-SNR outage probability expressions are studied and optimal power to be sent on wire is calculated analytically using outage probability expressions. Multi-traffic rate allocation and power allocation for a network are formulated as shown in Figure 1.1. Delay-sensitive data and voice should be sent efficiently through a wireless channel (fading channel) with additional mode of communication (wire). The problem is simplified as shown in Figure 1.2. 


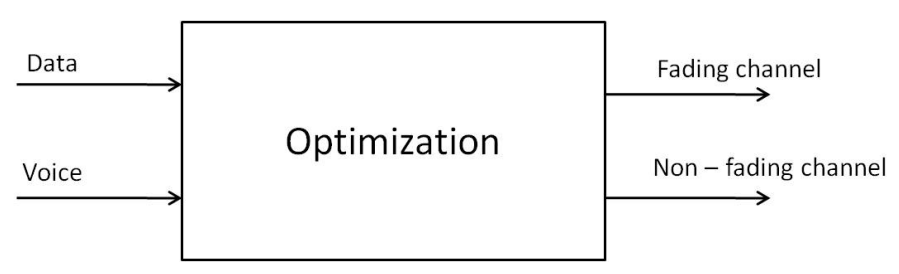

Figure 1.1: Block Diagram 1

First, the rate $R$ is divided between delay-sensitive data $\left(R_{d}\right)$ and voice $\left(R_{v}\right)$ to achieve

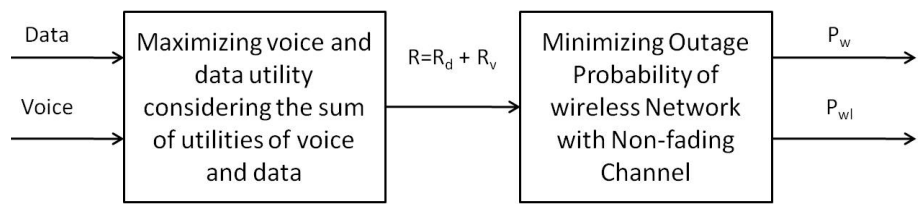

Figure 1.2: Block Diagram 2

maximum utility in the network. Utility function for the delay-sensitive data is considered from [13], a function of rate, delay and reliability and, the utility function for voice [26, 13] is a function of delay and reliability. These two utility functions are added together and total utility is maximized with respect to the constraint $R_{d}+R_{v}=R$. In the next step, the total transmit power $P$ is divided between the wireless channel $\left(P_{w l}\right)$ and the wired channel $\left(P_{w}\right)$ for a source-destination pair multimodal network and a cooperative diamond relay multimodal network separately using constrained optimization techniques. In the cooperative diamond relay multimodal network, wire is placed between nodes and signal is transmitted using distributed space time decode and forward protocol [21] to analyze outage probability expressions and minimum transmit power that should be sent through wire is calculated.

The thesis is organized as follows. Chapter 2 gives an overview of the wireless channel, small and large scale fading in wireless communication systems and cooperative diversity protocols used in cooperative communications. Chapter 3 contains rate allocation problem for delay-sensitive data and voice and results are presented. In Chapter 4, a source-destination pair multimodal network is studied and power allocation is done to achieve maximum instan- 
taneous rate and minimum outage probability in the network. In Chapter 5, a cooperative wireless relay network with a wire between nodes is considered and outage probability for the network implemented with space time decode and forward protocol is studied and plotted. Chapter 6 concludes with some insights to future work. 


\section{Chapter 2}

\section{Basic Concepts and Background}

\subsection{Wireless Communication Concepts}

\subsubsection{Transmitted and Received Signal Models}

The communication system propagates a signal from source to destination through a physical medium. Signals transmitted and received are considered to be real. We model communication systems using a complex frequency response for analytical simplicity. In fact, the channel just introduces an amplitude and phase change at each frequency of the transmitted signal so that the received signal is also real. Transmitted and received signals we use are the complex baseband representation of bandpass signals. The transmitted signal is modeled as [27]

$$
\begin{aligned}
s(t) & =\Re\left\{u(t) e^{j 2 \pi f_{c} t}\right\} \\
& =\Re\{u(t)\} \cos \left(2 \pi f_{c} t\right)-\Im\{u(t)\} \sin \left(2 \pi f_{c} t\right) \\
& =x(t) \cos \left(2 \pi f_{c} t\right)-y(t) \sin \left(2 \pi f_{c} t\right)
\end{aligned}
$$

where $u(t)=x(t)+i y(t)$ is a complex baseband signal with in-phase component $x(t)=$ $\Re\{u(t)\}$, quadrature component $y(t)=\Im\{u(t)\}$, bandwidth $B_{u}$ and power $2 P$. The signal $u(t)$ is called the complex envelope of $s(t)$. This is a standard representation of bandpass signals with $B<<f_{c}$, as it allows signal manipulation via $u(t)$ irrespective of the carrier frequency. The power in the transmitted signal $s(t)$ is $P$. The received signal is in the form 
$[27]$

$$
r(t)=\Re\left\{v(t) e^{j 2 \pi f_{c} t}\right\}
$$

where the complex bandpass signal $v(t)$ will depend on the channel which $s(t)$ propagates. If $s(t)$ is propagated through a time-invariant channel then $v(t)=u(t) * c(t), c(t)$ is the equivalent lowpass channel impulse response of the channel.

\subsubsection{Wireless Channel}

The transmission path between the transmitter and the receiver can vary from simple lineof-sight to one that is obstructed by buildings and mountains. Signal travels along multiple paths as it is reflected by various objects. At the receiver, these multipath components add constructively or destructively depending upon the attenuation factor and their phase angles causing the received signal strength to fluctuate with time and distance. The more practical wireless channel models consider the effects of fading which represents the fluctuations in the instantaneous received signal strength due to the multipath propagation of the transmitted signal.

Multipath fading can be considered as the significance difference between wired and wireless communications. The three basic propagation mechanisms that effect the signal propagation characteristics in the radio communication systems are reflection, diffraction and scattering. Reflection occurs when an EM wave strikes a smooth surface of an object having dimensions larger than the signal wavelength. Diffraction occurs when secondary waves form behind a dense body whose dimensions are large compared to $\lambda$. Scattering occurs when an EM wave strikes a large rough surface whose dimensions are in the order of $\lambda$. This causes reflected energy to spread out in all directions. These three propagation mechanisms depend on the amplitude, phase and polarization of the incident electromagnetic wave and also on the geometry of the object [28]. Large scale fading and small scale fading are two main types of fading which can be described by the above mentioned propagation mechanisms. 


\subsubsection{Small Scale Fading and Multipath}

Small scale fading is usually referred to simply as fading. It characterizes rapid fluctuations of amplitude, phase and multipath delays of a radio signal over a short period of time or travel distance, so that large-scale effects may be ignored. The resultant signal at the receiver is the sum of many contributions from different directions. Since the phases are random, the sum of contributions varies widely. In small scale fading, received signal may vary as much as three or four orders of magnitude when the receiver is moved by only a fraction of wavelength. Small scale fading can even occur at stationary receivers due to motion of surrounding objects in the radio channel. The four main factors effecting small scale fading are multipath propagation, speed of the mobile, speed of surrounding objects and transmission bandwidth of the signal. Relative motion between transmitter and receiver causes doppler shifts on each multipath component. Higher speeds result in larger doppler shifts, which are more difficult to deal with than small shifts.

\subsubsection{Rayleigh Fading Distribution}

Some probability distributions are used to model the received signal envelope which is determined by $\alpha_{n}(t)_{n}$. These models assume a large number of scatters so that the central limit theorem leads to a Gaussian model for the channel impulse response. Rayleigh distribution is used to describe the statistical time varying nature of the received envelope of a flat fading channel. It is used for a channel that do not have a strong line of sight. We know that the envelope of the sum of two quadrature Gaussian noise channels obeys a Rayleigh distribution. Dropping the path $n$ index we can write the fading coefficients as

$$
\alpha(t) e^{-j 2 \pi f_{c} \tau(t)}=h(t)=h_{r}(t)+j h_{i}(t)
$$

where $\alpha(t)=\sqrt{h_{r}^{2}(t)+h_{i}^{2}(t)}$ is the Rayleigh fading envelope and $h_{r}(t), h_{i}(t)$ are independent zero mean real random processes. If we assume large number of scatters, we can model $h_{r}(t), h_{i}(t)$ as i.i.d Gaussian using the central limit theorem. 


\section{Block Fading}

Block fading is one of the simplest time-variant fading models . The fading coefficients in block fading are modeled as constant over a block of symbols and vary independently between blocks. Consider a block of $N$ symbols, each with duration $T$. Then the baseband received signal in frequency non-selective block fading can be written as [29]

$$
r_{l}(t)=h s_{l}(t)+n(t), 0<t \leq N T
$$

where $s_{l}(t)$ is the baseband transmitted signal and $h \in \mathbb{C}$ is a random variable drawn, typically from a complex Gaussian distribution so that $|h|$ is Rayleigh. $h$ does not change during the block but changes during the second block $N T<t \leq 2 N T$. In this thesis, Rayleigh block fading is considered.

\subsubsection{Maximum Ratio Combining}

The main purpose of diversity is to coherently combine the independent fading paths so that the effects of fading are reduced. Combining is done before detection since the branch signal power or phase is required to determine the appropriate value of attenuation factor. Combining after detection requires a dedicated receiver for each branch to determine the phase which increases the hardware complexity and power consumption. Selection combining, equal ratio combining and maximum ratio combining are the most commonly used combining techniques. Maximum ratio combining (MRC) is superior to equal ratio combining and selection combining. In maximum ratio combining, signals from all $M$ branches are weighted according to their individual signal to noise ratios and then added. So, maximum signal to noise ratio is obtained at the receiver.

Assume i.i.d fading on each branch with equal branch SNR $\bar{\gamma}$. The average SNR at the receiver employing MRC technique is $\bar{\gamma}_{\Sigma}=M \bar{\gamma}$. The corresponding SNR outage probability ${ }^{1}$ for a given threshold $\gamma_{0}$ is given by [27]

$$
P_{\text {out }}=P\left(\gamma_{\Sigma}<\gamma_{0}\right)=1-e^{\gamma_{0} / \bar{\gamma}} \sum_{k=1}^{M} \frac{\left(\gamma_{0} / \bar{\gamma}\right)^{k-1}}{(k-1) !} .
$$

\footnotetext{
${ }^{1}$ Probability that the average SNR is less than threshold SNR $\gamma_{0}$ is SNR outage probability and is given by $P_{\text {out }}=P\left(\gamma_{\sum}<\gamma_{0}\right)$.
} 


\subsubsection{Space Time Coding}

Space time coding is a powerful transmit diversity technique that relies on coding across space and time to extract diversity. Space time block coding was introduced in $[30,31]$ which generalizes the transmission scheme discovered by [32].

The Alamouti space time block code uses simple ML decoding with two transmit antennas and number of receiving antennas varies with the code construction. This scheme works over two symbol periods where the channel gain is constant over time. Let signal $s_{1}$ transmitted by antenna 1 and $s_{2}$ transmitted by antenna 2 over the first symbol period each with symbol energy $\frac{E_{s}}{2}$. Over the next symbol period $-s_{2}^{*}$ is transmitted from the antenna 1 and $s_{1}^{*}$ from the antenna 2 , each with symbol energy $\frac{E_{s}}{2}$. So the space time block code can be represented as

$$
\left[\begin{array}{cc}
s_{1} & s_{2} \\
-s_{2}^{*} & s_{1}^{*}
\end{array}\right] .
$$

The receiver symbol over the first symbol period is $y_{1}=h_{1} s_{1}+h_{2} s_{2}+n_{1}$ and the received symbol over the second symbol period is $y_{2}=-h_{1} s_{2}^{*}+h_{2} s_{1}^{*}+n_{2}$, where $n_{i}, i=1,2$ is the AWGN noise sample at the receiver related with $i$ th symbol transmission. So the receiver uses these sequentially received symbols to form the vector $\mathbf{y}=\left[\begin{array}{ll}y_{1} & y_{2}^{*}\end{array}\right]^{T}$ given by [27 $]$

$$
\mathbf{y}=\left[\begin{array}{cc}
h_{1} & h_{2} \\
h_{2}^{*} & -h_{1}^{*}
\end{array}\right]\left[\begin{array}{l}
s_{1} \\
s_{2}
\end{array}\right]+\left[\begin{array}{l}
n_{1} \\
n_{2}^{*}
\end{array}\right]=\mathbf{H}_{A} s+\mathbf{n},
$$

where $\mathbf{s}=\left[\begin{array}{ll}s_{1} & s_{2}\end{array}\right]^{T}, \mathbf{n}=\left[\begin{array}{ll}n_{1} & n_{2}\end{array}\right]^{T}$ and

$$
\left[\begin{array}{cc}
h_{1} & h_{2} \\
h_{2}^{*} & -h_{1}^{*}
\end{array}\right] .
$$

If we find a new vector $\mathbf{z}=\mathbf{H}_{A}^{H} \mathbf{y}$ then

$$
\mathbf{z}=\left[\begin{array}{ll}
z_{1} & z_{2}
\end{array}\right]^{T}=\left(\left|h_{1}^{2}\right|+\left|h_{2}^{2}\right|\right) \mathbf{I}_{2} s+\tilde{\mathbf{n}}
$$

where $\tilde{\mathbf{n}}=\mathbf{H}_{A}^{H} \mathbf{n}$ is a complex Gaussian noise vector. The diagonal nature of signal portion of $\mathbf{z}$ effectively decouples the two symbol transmissions, so that the each component of $\mathbf{z}$ corresponds to one of the transmitted symbols

$$
z_{i}=\left(\left|h_{1}^{2}\right|+\left|h_{2}^{2}\right|\right) s_{i}+\tilde{n}_{i}, i=1,2 .
$$


Thus the received SNR is

$$
\gamma_{i}=\frac{\left(\left|h_{1}^{2}\right|+\left|h_{2}^{2}\right|\right) E_{s}}{2 N_{0}}
$$

where the factor 2 comes from the fact that $s_{i}$ is transmitted with total symbol energy

$\frac{E_{s}}{2}$. The received SNR is equal to the sum of SNRs of each branch. The diversity gain of STBC is same as two branch MRC but there is coding loss of $3 \mathrm{~dB}$ because because of the simultaneous transmission of two distinct symbols from two antennas [32]. Alamouti's scheme works only for two transmit antenna case. The Alamouti scheme can be generalized for more than two transmit antennas when the constellations are real [27].

\subsection{Capacity and Outage Probability}

\subsubsection{Channel Capacity}

Channel capacity was first developed by Shannon [33], as a mathematical theory of communication based on the notion of mutual information between input and output of the channel. Capacity is defined as the number of bits of information that can be transmitted with arbitrarily low error probability over a channel. Capacity limits gives the maximum data rates that can be transmitted over a wireless channel with small error probability and assuming no constraints on delay or complexity of the encoder and decoder [27]. Shannon's information channel capacity is given by [33]

$$
C=B \log _{2}\left(1+\frac{P}{B N_{o}}\right) \text { bits/second. }
$$

where $B$ is bandwidth, $P$ is continuous-time transmit power in Watts and $N_{0}$ is the Gaussian noise of the channel

\subsubsection{Capacity in an Additive White Gaussian Noise Channel}

Consider a discrete-time additive white Gaussian noise (AWGN) channel with channel input/output relationship $y[i]=h x[i]+n[i]$, where $x[i]$ is the channel input at time $i, y[i]$ is the corresponding channel output, and $n[i]$ is the white Gaussian noise random process. Assume channel bandwidth $B$, transmit power $P$ and channel gain $h$ in the channel. The 
SNR of the channel is defined using the symbol SNR ${ }^{2}$ as $\frac{P}{N_{0} B}$. The capacity of the AWGN channel is given by $[27,33]$

$$
\begin{aligned}
C & =B \log _{2}\left(1+|h|^{2} \mathrm{SNR}\right) \\
& =B \log _{2}\left(1+\frac{|h|^{2} P}{N_{0} B}\right) \mathrm{bits} / \mathrm{sec}
\end{aligned}
$$

where $N_{0}$ is the noise spectral density of the channel. Figure 2.1 shows the capacity plot for AWGN channel. Capacity of the channel increases with increase in SNR, but saturates at a point. Then, increase in bandwidth might increase the capacity of the channel.

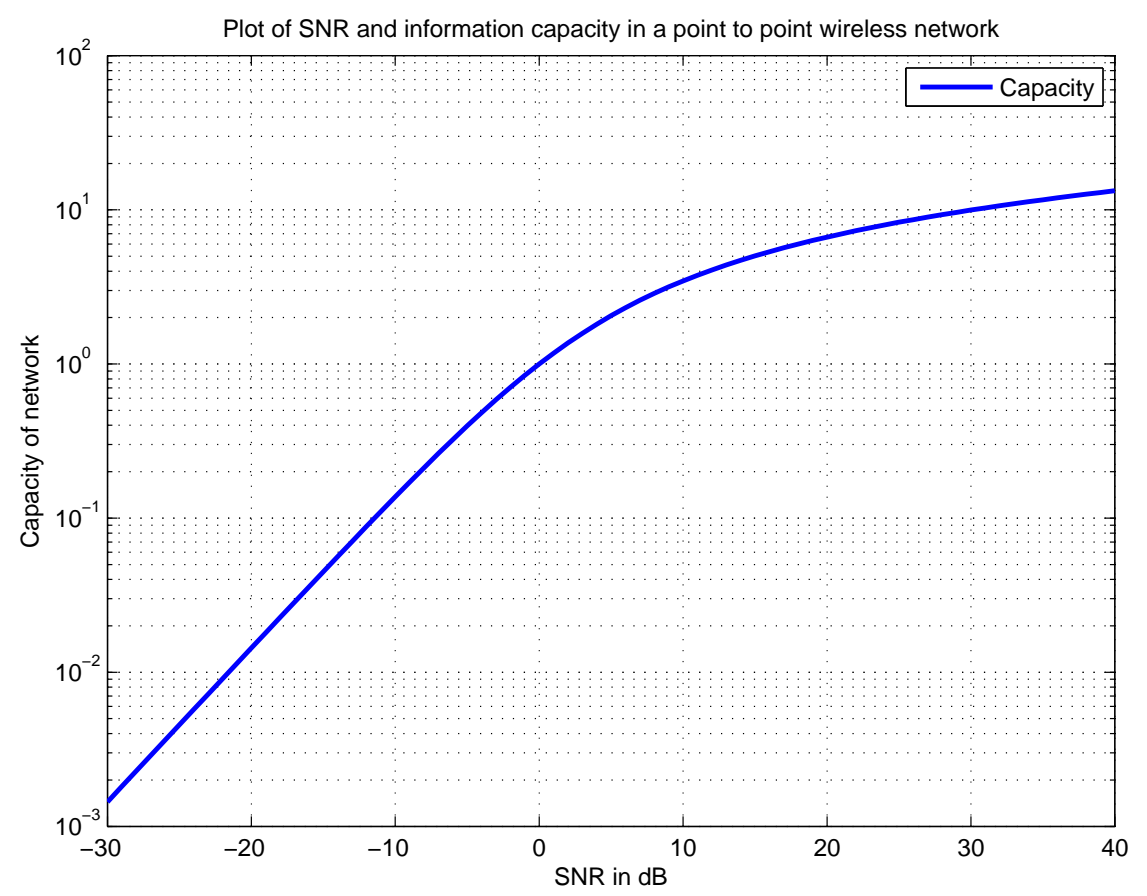

Figure 2.1: Capacity plot for a AWGN channel with increase in SNR

\subsubsection{Capacity for Fading Channels}

Consider a discrete-time channel which undergoes Rayleigh fading, with noise power $N_{0} B$

$$
r[i]=h[i] s[i]+n[i]
$$

\footnotetext{
${ }^{2} \mathrm{SNR}=\frac{\text { signal power }}{\text { noise power }}=\frac{P}{N_{0} B}$ where, $P$ is the the continuous-time transmit signal power, $B$ is the signal bandwidth, $N_{0}$ is the noise spectral density of the channel and $h$ is the channel gain.
} 
where $h[i]$ is random. For this type of fading channel,

$$
C=B \log _{2}\left(1+\frac{|h[i]|^{2} P}{N_{0} B}\right) \text { bits/sec }
$$

where $P$ is the transmit power, $B$ is the bandwidth of the channel, $h[i]$ is the fading coefficient at time $i$ and $N_{0}$ is the noise spectral density of the channel. Equation (2.18) is a random variable and does not represent capacity of the channel. The capacity of the channel is the average over the distribution $h[i]$ and is termed as ergodic capacity of the channel.

Ergodic capacity indicates the largest rate that is achievable with low error rate. Ergodic capacity of a fading channel is found by averaging the instantaneous capacity over the fading coefficient distribution. But we need the block length that is enough to experience many different channel conditions. In slow fading, the channel code block length may be short that each code word experiences very few channel realization. In this case, it is impossible to code for arbitrarily low error probability. In such cases we can't calculate the exact capacity of the network.

\subsubsection{Information Outage Probability for Non-ergodic Channel}

Capacity is not a good way to analyze the performance of slow fading channels. Instead the performance of slow fading channels is calculated by looking at the probability that the instantaneous achievable rate will be less than a given rate $R$. This is called as information outage probability and is defined as [29]

$$
P_{\text {out }}(R) \triangleq P_{r}\left[\log _{2}\left(1+\frac{P|h|^{2}}{N_{0} B}\right)<R\right] .
$$

Assume Rayleigh fading channel where $r[i]=h[i] s[i]+n[i]$ is receive signal. The outage for this channel becomes

$$
\begin{aligned}
P_{\text {out }}(R) & \triangleq P_{r}\left[\log _{2}\left(1+|h|^{2} \mathrm{SNR}\right)<R\right] \\
& =1-e^{\frac{-\left(2^{R}-1\right)}{\mathrm{SNR}}}
\end{aligned}
$$

where the random variable $|h|^{2}$ has an exponential distribution. At high SNR, we can approximate (2.21) using a Taylor series expansion as

$$
P_{\text {out }}(R) \approx \frac{2^{R}-1}{\mathrm{SNR}}
$$


So the outage probability decreases at the rate $\frac{1}{\mathrm{SNR}}$. Fig 2.2 shows the exact and high SNR outage probability plot for a Rayleigh fading channel.

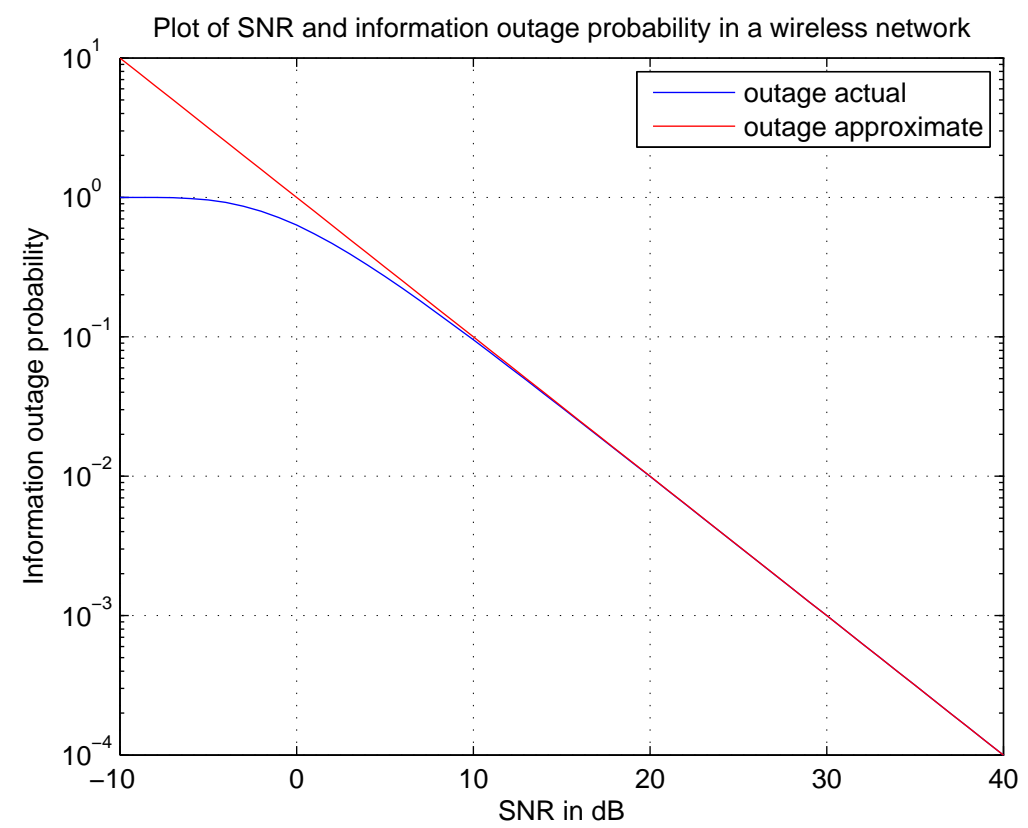

Figure 2.2: Information outage probability plot for a AWGN channel with increase in SNR

\subsection{Resource Allocation for Data and Voice}

Wireless services integrates different types of traffic, such as voice, data, images, videos, etc. Because these traffic streams have different requirements on rate and performance, allocation of scarce resources such as power, rate and bandwidth can have a significance effect on the system performance. In Chapter 3, we study resource allocation for voice and data traffic in multimodal networks. Voice and data have different rate, delay, reliability requirements. Voice packets can tolerate errors and even small packet loss while data packets are sensitive to loss and errors but can tolerate delay. So, in an integrated voice and data network, priority should be given to voice packets to limit their delays. There are many ways to control the performance of the system associated with different data streams. The resource allocation problem can be formulated as a constrained optimization of an objective function in power and rate so that minimum power is used to achieve maximum rate for 
data and voice users. In [4], a movable boundary method is used to integrate voice and data networks. The channel is partitioned into frames with a fixed length. Voice and data are integrated using a movable boundary TDMA. A frame is divided into two regions with a boundary between them. The first region is used for voice and data with voice having high priority while second region is used only for data packets. The boundary moves in accordance with the number of active voice packets in each frame. In this way, data and voice traffic are separated and transmitted. System performance was analyzed considering throughput and delay parameters varying the number of users. These are some methods introduced to allocate resources in voice and data networks.

Network performance should be evaluated in terms of the degree to which network satisfies the users' requirements rather than system parameters like outage probability, throughput, etc. When a user is assigned certain resources utility theory provides a means to formulate the gain of a quality measurement. System performance and efficiency of resource allocation can be measured by the achieved network utility or fairness. In this thesis, utility functions are considered to measure the level of users' satisfaction and total system utility as an optimization objective to solve the traffic optimization problem. Traffic optimization model in this thesis is motivation from the work in [11] and [13] which has the same objective of maximizing aggregate utility. In [11], two different classes of rate control algorithms are introduced considering utility functions in terms of rate only. As it does not reflect all the user requirements, utility function in terms of rate, delay and reliability should be considered. In [13], capacity is allocated to mixture of traffic types, including VoIP, delay sensitive and delay-insensitive data traffic considering appropriate rate-reliability characteristics and maximizing their combined utility in a network with composite links. The optimization problem here is a non-convex optimization problem and it is approximated as convex problem.

\subsubsection{Utility Function}

Utility functions can be used to quantify the performance of a network as a function of data rate, delay, power, bandwidth, etc. They play a key role in resource management and QoS differentiation. Utility functions vary for applications based on their requirements. 
They are generally designed using two different approaches. One is by complex subjective surveys and other is based on the habits of the traffic and appropriate fairness in the network [34]. $(U(\cdot))$ can be used to represent utility function. Utility functions must be designed so that they satisfy all the user requirements. Generally they are in terms of rate, delay, reliability. If we consider three types of traffic: voice traffic (e.g.,VoIP), utility is a function of delay and packet loss; delay-insensitive traffic, where utility is a function of rate and reliability; and delay-sensitive traffic, where utility depends on rate, reliability and delay. In general, there are three different types of utility functions.

1. Concave utility functions

2. Sigmoidal utility functions

3. Convex utility functions

\subsection{Cooperative Communications}

The classical relay channel models were first introduced by van der Meulen [35, 36]. They are generally called relays which receive, process and re-transmit some information in order to improve performance of the system. In [20], capacities of gaussian relay channels and certain discrete relay channels are evaluated. Cover and El Gamal [20] studied non-faded relay channel models and developed lower and upper bounds on the channel capacity and developed a cooperation scheme in which the relay completely decodes the source message and retransmits some information in the signal to the destination. The destination suitably combines the source and relay transmissions to achieve higher rates compared to the direct transmission.

Cooperative communications considers the participation of several relay nodes (in addition to the source node) in delivering a signal to the destination node to achieve diversity gain. Relay channels setup diversity gains by sending additional copies of the signal through relays. In [22], a variety of low-complexity, cooperative protocols that exploit the broadcast and spatial diversity of the channel are developed and amplify and forward and decode and forward algorithms are introduced. In [21], Laneman et al. proposed and analyzed different 
space-time cooperative diversity protocols for cooperative relay networks. A medium access control protocol where time orthogonal transmissions between source-transmission and relaydestination is considered which is a limitation in their work. These protocols are studied in this section as they are used in this thesis.

Consider a wireless network as in [21] with a set of transmitting terminals denoted by $M=1,2, \ldots, M$. The source $s \in M$ transmits information to destination $d(s) \notin M$ using $M-s$ relays. In cooperative diversity algorithms, decoding set $D(s)$, is the set of relays that can decode and forward the message to the destination. Signal transmission is divided into two phases. During the first phase each potential relay $r \in M-s$ receives [22]

$$
y_{r}[n]=a_{s, r} x_{s}[n]+z_{r[n]}
$$

where $x_{s}[n]$ is the source transmitted signal and $y_{r}[n]$ is the received signal at $r$. For decode and forward transmission, if the SNR is sufficiently large for $r$ to decode the signal from source, then $r$ serves as decoding relay for the source $s$, so that $r \in D(s)$. The destination $d(s)$ receives the signals in both the phases. During the first phase from source,

$$
y_{d(s)}[n]=a_{s, d(s)} x_{s}[n]+z_{d(s)}[n] .
$$

During the second phase, the destination receives the signal from all the relays in the decoding set for space-time-coded cooperative diversity. Those signal transmissions occur in the same subchannel are superimposed at the destination, so that

$$
y_{d(s)}[n]=\sum_{r \in D(s)} a_{r, d(s)} x_{r}[n]+z_{d(s)} .
$$

For repetition-based cooperative diversity, the destination receives separate transmissions from each of the relays, i.e., for $r \in M-s$, we model the received signal $d(s)$ as

$$
y_{d(s)}[n]=a_{r, d(s)} x_{r}[n]+z_{d(s)}
$$

in the appropriate channel, where $x_{r}[n]$ is the transmitted signal of relay $r$. In (2.23) - (2.26), the magnitudes $\left|a_{s, r}\right|,\left|a_{r, d(s)}\right|$ and $\left|a_{s, d(s)}\right|$ are channel gains which are Rayleigh distributed and include the effects of pathloss, shadowing and fading. $1 / \lambda_{s, r}, 1 / \lambda_{r, d(s)}$ and $1 / \lambda_{s, d(s)}$ are 
variances of the corresponding magnitudes of channel gains. $z_{r[n]}, z_{d(s)}[n]$ and $z_{d(s)}$ are zeromean complex Gaussian random variables that capture the effects of receiver noise and other interferences in the system.

\subsubsection{Cooperative Diversity Protocols}

In this section, some cooperative diversity protocols used in relay networks are studied. These protocols employ different types of processing by the relay terminals and different types of combining at destination terminals.

\section{Amplify and Forward}

This method is used when the relay has a time or power constraint to decode and encode a message. In amplify and forward (AAF), the relay attenuates and amplifies the signal before sending it again. The incoming signal is amplified block-wise. The main disadvantage of this method is noise in the signal also gets amplified. Consider the channel model in (2.23) - (2.26). The source terminal transmits its information as $x_{s}[n]$ for $n=1,2 \ldots N / 4$, where $N$ consecutive uses of the channel are considered. During this interval, the relay processes $y_{r}[n]$ and relays the information by transmitting [22]

$$
x_{r}[n]=\beta y_{r}[n-N / 4]
$$

for $n=N / 4+1, \ldots, N / 2$. The relay must amplify the signal to sent it with the same power as the sender. So the amplifying gain is

$$
\beta \leqslant \sqrt{\frac{P}{\left|a_{s, r}\right|^{2} P+N_{0}}}
$$

Amplifier gain is depended on the fading coefficient $a_{s, r}$ between the source and relay. AAF protocol is viewed as the repetition coding from two separate transmitters where the relay amplifies its own noise. The AAF protocol just forwards the corrupted signal. So the bit error rate at the destination is very large. 


\section{Decode and Forward}

Decode and Forward (DAF) protocol is the most commonly used protocol where relay has sufficient power to decode the message. The received signal is decoded and encoded. So, no amplified noise is present in the signal sent as in AAF protocol. Consider the channel model in $(2.23)-(2.26)$. The source transmits the information $x_{n}[s]$ for $n=0, \ldots, N / 4$. During this interval, the relay processes $y_{r}[n]$ by decoding an estimate $\hat{x}_{s}$ of the transmitted signal. Under the repetition code scheme, the relay transmits the signal [22]

$$
x_{r}[n]=\hat{x}_{s}[n-N / 4]
$$

for $n=N / 4+1, \ldots, N / 2$. A relay might fully decode i.e estimate without error or it can do symbol by symbol decoding and allows the destination to perform full decoding.

\subsubsection{Laneman's Repetition-Based Cooperative Diversity Proto- col}

This protocol consists of a source that broadcasts message to the destination and potential delays. Those potential relays that can decode the message become decoding relays and they repeat the source message on the orthogonal subchannels. The relays employ the same codebook as the source which is complex Gaussian codebook in this network model. The transmission between the source and destination uses a fraction $1 / m$ degrees of freedom in the channel. Assuming $D(s)$ as the decoding set the mutual information between source $s$ and destination $d(s)$ is given by [21]

$$
I_{r e p}=\frac{1}{m} \log \left(1+\mathrm{SNR}\left|a_{s, d}\right|^{2}+\mathrm{SNR} \sum_{r \in D(s)}\left|a_{r, d}\right|^{2}\right)
$$

The mutual information between source and relay is given by

$$
\frac{1}{m} \log \left(1+\mathrm{SNR}\left|a_{s, r}\right|^{2}\right) .
$$

The outage probability expression using high-SNR approximations is given by

$$
\operatorname{Pr}\left[I_{r e p}<R\right] \sim\left[\frac{2^{m R}-1}{\mathrm{SNR}}\right]^{m} \times \sum_{D(s)} \lambda_{s, d} \times \prod_{r \in D(s)} \lambda_{r, d} \prod_{r \notin D(s)} \lambda_{s, r} \times \frac{1}{|D(s)+1| !} .
$$




\subsubsection{Laneman's Space-Time Coded Cooperative Diversity Pro- tocol}

Laneman's space-time coded cooperative diversity protocol consist of a source broadcasting its transmission to the destination and potential relays. The relays that can decode the transmission become decoding relays and transmit the signal simultaneously in the same subchannel using a suitable space-time code. This protocol has better bandwidth efficiency than repetition-based as the relays uses same subchannel. Each cooperation terminal transmits in $1 / 2$ the total degrees of freedom in the channel as there are two parallel channels, one from source to destination and other from decoding relays to destination. So the mutual information between source and destination on decoding set $D(s)$ is

$$
I_{s t c}=\frac{1}{2} \log \left(1+\frac{2}{m} \mathrm{SNR}\left|a_{s, d}\right|^{2}\right)+\frac{1}{2} \log \left(1+\frac{2}{m} \mathrm{SNR} \sum_{r \in D(s)}\left|a_{r, d}\right|^{2}\right)
$$

The expression for outage probability using high-SNR approximations is obtained as [21]

$$
\operatorname{Pr}\left[I_{s t c}<R\right] \sim\left[\frac{2^{2 R}-1}{2 \mathrm{SNR} / m}\right]^{m} \times \sum_{D(s)} \lambda_{s, d} \times \prod_{r \in D(s)} \lambda_{r, d} \prod_{r \notin D(s)} \lambda_{s, r} \times A_{|D(s)|}\left(2^{2 R}-1\right)
$$

where

$$
A_{n}(t)=\frac{1}{(n-1) !} \int_{0}^{1} \frac{w^{n-1}(1-w)}{(1+t w)} \mathrm{d} w .
$$

Equations (2.32) and (2.34) achieve full spatial diversity in the number of cooperating terminals, not just the number of decoding relays. These space-time coded cooperative diversity protocols achieve higher diversity order than repetition-based schemes for larger spectral efficiencies.

\subsection{Multimodal Networks}

A lot of research has been done on capacity limits of wireless networks under some assumptions on the physics of propagation and some restrictions on the communication strategy employed by the nodes. Gupta et al. [23] analyzed capacity of a wireless network with $n$ nodes located in a region of area $1 \mathrm{~m}^{2}$. It can been seen that the rate per user drops to 
zero as the number of users increases in that fixed area. In [24], it is proven that the capacity limits are due to a degree of freedom limitation related to fundamental electromagnetic laws of physics. From these works it can be concluded that very large high-performance purely wireless networks are not just difficult to design, but are actually impossible to build. In such cases, an additional mode of communication like wire solves the problem and decreases the rate burden on wireless channels. The advantage of wires is that they are non-fading and they do not contribute to the large-network bottleneck that drives throughput to zero. Wired networks offer high capacity and reliability compared to wireless networks. A wireless network with wire between nodes is termed as multimodal network in this thesis.

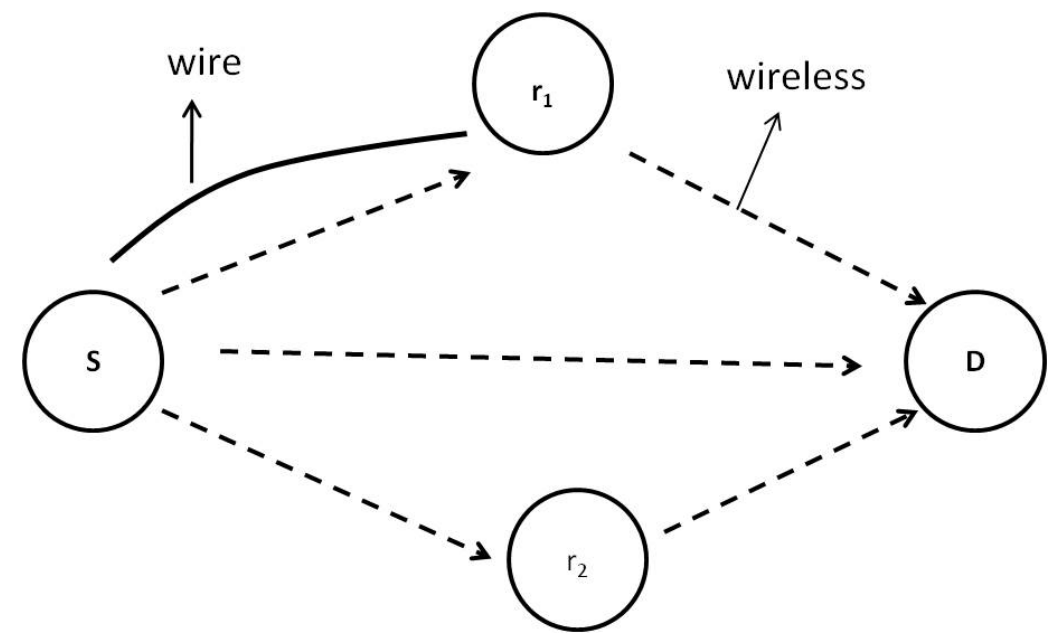

Figure 2.3: Multimodal Network

Consider a multimodal relay network as shown in the Figure 2.3. A wire is placed between the source $S$ and relay $r_{1}$. The addition of the wire can reduce outage probability at relay $r_{1}$. But performance of relay $r_{2}$ decreases as the power allocated to wireless channel is lowered. So, optimal power allocation should be done to wired and wireless channel to achieve best results. Research has been focused on optimal power allocation when two modes of communication that operate simultaneously are considered in relay networks.

In [25], a cooperative diamond wireless relay network with nodes having the capability to communicate through wires is considered. It is a jointly optimized multimodal communica- 
tion network with wired and wireless modes operating simultaneously. The relay network is implemented with an appropriately modified Laneman space time diversity protocol. HighSNR outage probability expressions are developed for two different network topologies. One is with wire between source and relay and other is with wire between relay and destination. Those expressions are similar to the outage probability expression (2.34) and are given below. Outage probability expression for the network with wire between source and relay is given by [25]

$$
\begin{aligned}
P_{r}[I<R] \approx & {\left[\frac{2^{2 R}-1}{2 \mathrm{SNR} / m}\right] \times \sum_{D(s)}\left[\frac{1}{1-P_{w}}\right]^{m-|D(s)|-1} } \\
& \times \lambda_{s, d(s)} \prod_{r \notin D(s)} \lambda_{s, r} \prod_{r \in D(s)} \lambda_{r, d(s)} \times A_{D(s)}\left(2^{2 R}-1\right)
\end{aligned}
$$

where $\lambda_{s, r}=\frac{\lambda_{s, r_{\text {orig }}}\left(2^{2 \hat{R}}-1\right)}{2^{2 R}-1}$ for wired relay, $\hat{R}=R-\frac{1}{2} \frac{B_{w}}{B_{w l}} \log \left(1+\frac{2 P_{w}}{m B_{w} / B_{w l} \mathrm{SNR}|h|^{2}}\right)$ and $\lambda_{s, r_{\text {orig }}}=$ $\lambda_{s, r}$. Outage probability expression for the network with wire between relay and destination is given by $[25]$

$$
\begin{aligned}
P_{r}(I<R) \approx & {\left.\left[\frac{1}{2 \mathrm{SNR} / m}\right]^{m} \times \lambda_{s, d(s)}\right) \times\left[\left(\frac{\lambda_{r_{w}, d(s)}}{\left(1-P_{w}\right)}\right.\right.} \\
& \times \sum_{D(s): r_{w} \in D(s)} \times\left(2^{2 \hat{R}}-1\right)^{|D(s)|+1} \times\left(2^{2 R}-1\right)^{m-|D(s)|-1} \\
& \left.\times \prod_{r \in D(s): r \neq r_{w}} \lambda_{r, d(s)} \prod_{r \notin D(s)} \lambda_{s, r} \times A_{|D(s)|}\left(2^{2 \hat{R}}-1\right)\right) \\
& \left.+\left(\left(2^{2 R}-1\right)^{m} \times \lambda_{s, r_{w}} \times \sum_{D(s): r_{w} \notin D(s)} \lambda_{s, r} \times A_{|D(s)|}\left(2^{2 R}-1\right)\right)\right] . \\
& \times \prod_{r \in D(s)} \lambda_{r, d(s)} \prod_{r \notin D(s): r \neq r_{w}}
\end{aligned}
$$

Expressions (2.35) and (2.36) show that the multimodal space-time cooperative relay network also achieve full diversity as Laneman space time cooperative relay network. Outage probability curves for source-relay and relay-destination network models are duplicated in Figures 2.4 and 2.5 with equal bandwidths for both modes. The additional mode wire is placed between the nodes where wireless channel is worst. In source-relay case, the optimal power allocation should be done at the power where wire can support at least target rate $R$. If more power is allocated to wire the decoding capacity of other relay decreases which results 
in the increase in outage probability. From the plots it is observed that the performance of the network is increased when wire is placed in any of those two configurations and wire between source and relay has better performance compared to wire between relay and destination.

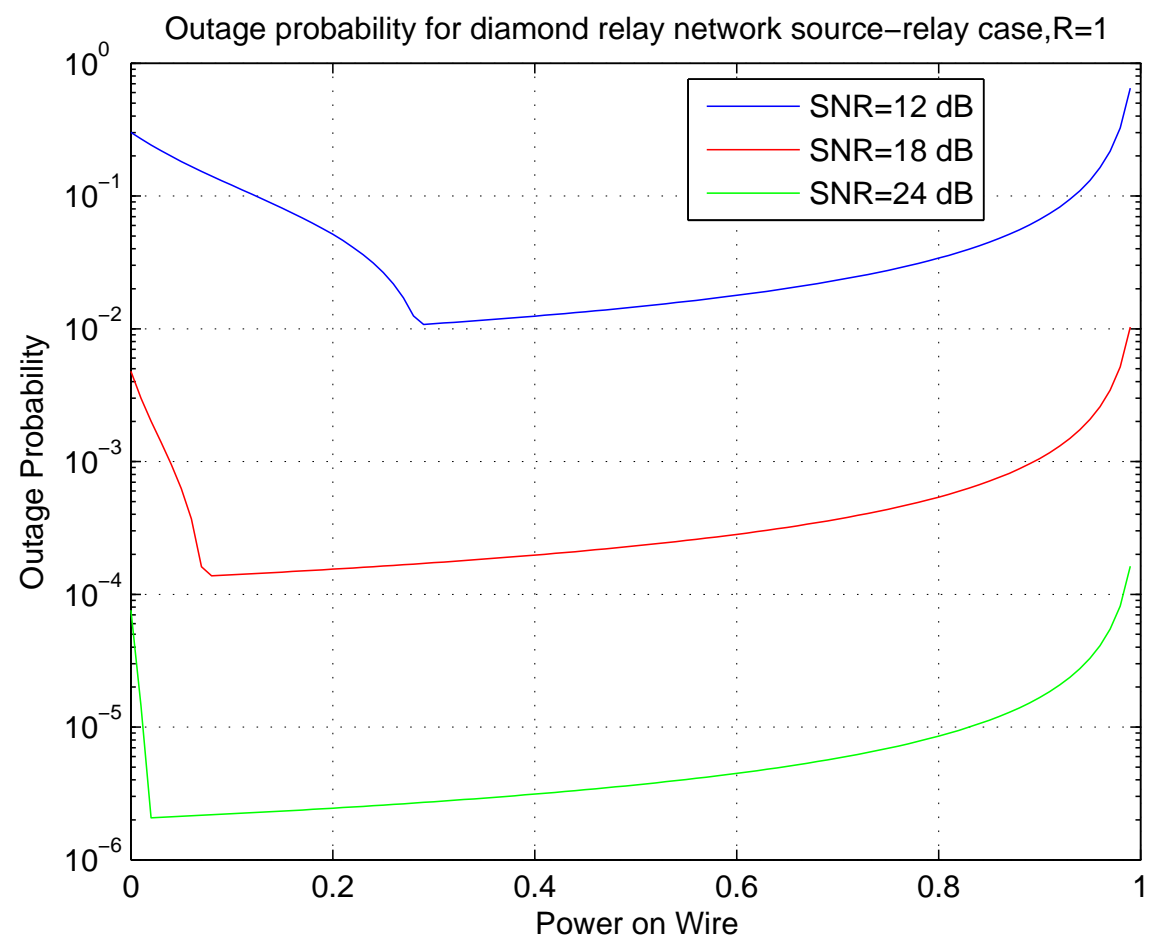

Figure 2.4: Outage probability curves for diamond relay network with wire between source and relay for three different values of SNR

\subsection{Summary}

The practical wireless channels should consider the effects of fading and noise. Cooperative diversity protocols can be used to mitigate the effects of fading due to multipath propagation in wireless networks. AAF and DAF are low-complexity cooperative diversity protocols which are widely used. Space-time coded protocols provides an efficient way to exploit full spatial diversity for more than two relays in the network. Efficiency of resource allocation for voice and data can me measured by the achieved network utility. The performance of cooperative wireless networks can be increased by introducing an additional mode of communication like wire as it decreases the outage probability of the network. 


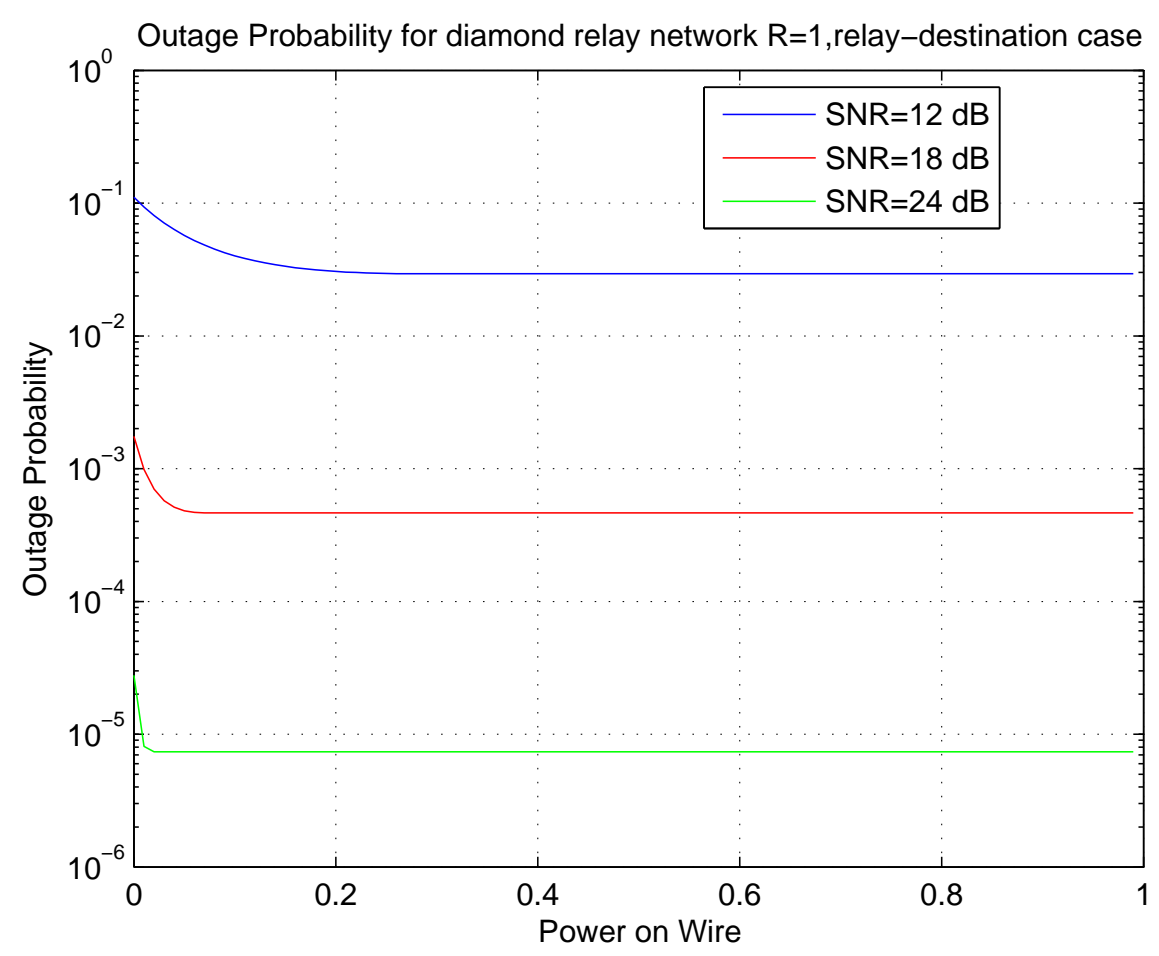

Figure 2.5: Outage probability curves for diamond relay network with wire between relay and destination for three different values of SNR 


\section{Chapter 3}

\section{Traffic optimization in Heterogeneous Traffic Networks}

Most advanced cellular networks and WLANs need to support multimedia services with high data rates. Resource allocation and traffic optimization are crucial problems in multitraffic networks as resources are scarce and traffic is shared by multiple users. For a whole network, the performance can be evaluated in terms of the degree to which it satisfies the service requirements of users' applications [38] rather than other system-centric quantities like throughput, outage probability and power etc. When application performance is the key concern in a network, utility could be considered as a reliable metric. Utility and optimization based resource allocation problems have been studied by many researchers. These are helpful to design and control wireless and wire-line networks. Kelly et al. [11] analyzed different rate control algorithms based on utility functions for a network model and introduced the framework of Network Utility Maximization (NUM) that has many applications in network resource allocation algorithms. Using utility functions, traffic optimization problems are formulated into convex or non-convex optimization problems and solved using Lagrange multipliers and Kuhn-Tucker theorem.

\footnotetext{
${ }^{1}$ Part of the work presented in this chapter appears in [37].
} 


\subsection{Network Model}

In this thesis, we consider a heterogeneous network with delay-sensitive data and voice traffic. Assume two sources that transmit voice and delay-sensitive data packets with packet size $K$ bits. We also assume that the network can differentiate voice and data. The term data refers to delay sensitive data in later part of the thesis. Let $R_{d}$ be the rate allocated to data and $R_{v}$ be the rate allocated to voice. The rate $R$ should be shared by the data and voice traffic such that $R=R_{d}+R_{v}$. We assume incoming data and voice packets are stored in separate queues and transmitted in a first-in-first-out (FIFO) fashion. The Kleinrock independence approximation [39] can be applied to a queue where packets need to wait for their transmission. According to the Kleinrock independence approximation, the average delay for the queues as mentioned above can be approximately calculated by assuming that the delays in the queues are independent. Applying Kleinrock independence approximation, data and voice queue can be modeled as a $M / D / 1$ queue $^{1}$ and delay is calculated accordingly. The average end to end delay for the data source is $\delta_{d}$ and for the voice source is $\delta_{v}$ and units for $\delta_{v}$ and $\delta_{d}$ are sec/wireless $\mathrm{Hz}$ and permitted range of values are from 0 to 200 millsec/wireless $\mathrm{Hz}$. The end to end delay refers to transmission and queuing delays. $\rho_{d}=1-p_{d}$ and $\rho_{v}=1-p_{v}$ are reliabilities for data source and voice source respectively, where $p_{d}$ and $p_{v}$ are end-to-end packet error probabilities for the data and voice. Traffic optimization is based on maximizing the sum of utilities of all voice and data users. Utility functions for data and voice are defined in the next section.

\subsubsection{Utility Function for Data}

We consider utility function for delay-sensitive data as a function of rate, reliability and delay and is represented as $U_{d}\left(R_{d}, \rho_{d}, \delta_{d}\right)$. It can be a weighted sum of utility on delay and throughput with the weight $w_{d} \in[0,1]$ indicating the relative importance of throughput and

\footnotetext{
${ }^{1}$ To calculate delay in queues the arrival and departure of packets from queues are considered as some probability distributions. $M / D / 1$ is a queue notation which states that the data and voice packets arrive into the queue as a deterministic distribution denoted as $M$ and depart the queue as an exponential distribution denoted as $D$ and can come out of the queue through one exit [2].
} 
delay $[13]$

$$
U_{d}\left(R_{d}, \rho_{d}, \delta_{d}\right)=w_{d} \frac{R_{d} \rho_{d}-\left(r_{d} \rho_{d}\right)^{\min }}{\left(r_{d} \rho_{d}\right)^{\max }-\left(r_{d} \rho_{d}\right)^{\min }}-\left(1-w_{d}\right) \frac{\delta_{d}-\delta_{d}^{\min }}{\delta_{d}^{\max }-\delta_{d}^{\min }}
$$

The average packet delay, $\delta_{d}$ is calculated for the data source by assuming the Kleinrock independence approximation and is given by $[2,39,13]$

$$
\delta_{d}=\frac{K}{2}\left(\frac{1}{R}+\frac{1}{R-R_{d}}\right)
$$

where $K$ is the packet size. The data utility $U_{d}\left(R_{d}, \rho_{d}, \delta_{d}\right)$ is a function that has relative importance of throughput and delay by placing a weight $w_{d}$. After substituting $\delta_{d}$ in (3.1)

$$
U_{d}\left(R_{d}, \rho_{d}, \delta_{d}\right)=w_{d} \frac{R_{d} \rho_{d}-\left(r_{d} \rho_{d}\right)^{\text {min }}}{\left(r_{d} \rho_{d}\right)^{\text {max }}-\left(r_{d} \rho_{d}\right)^{\min }}-\left(1-w_{d}\right) \frac{\frac{K}{2}\left(\frac{1}{R}+\frac{1}{R-R_{d}}\right)-\delta_{d}^{\text {min }}}{\delta_{d}^{\max }-\delta_{d}^{\min }} .
$$

\subsubsection{Utility Function for Voice}

\section{$R$-factor}

The Rating factor ( $R$-factor) is the output from the E-model, an International Telecommunication Union (ITU) transmission rating model [26] used to ensure that the users are satisfied with the end-to-end transmission performance of voice. The E-model estimates the conversational quality from mouth to ear as perceived by the user at the receiver side. The rating factor $R^{f a c}$ is composed of [26]

$$
R^{f a c}=R_{o}-I_{s}-I_{d}-I_{e}+A
$$

where $R_{o}$ represents the basic SNR, $I_{s}$ represents the combination of all impairments which occur more or less simultaneously with the voice signal, $I_{d}$ represents the impairments caused by the delay, $I_{e}$ represents impairments caused by low bit rate $\operatorname{codecs}^{2}$ and $A$ is the advantage factor that corresponds to the user allowance due to the convenience in using a given technology. The parameters $R_{o}, I_{s}$ and $I_{d}$ are subdivided into further specific impairment values. The E-model provides a statistical estimation of quality measures. So, the $R$-factor for voice can be considered as the utility function for voice. The $R$-factor and voice quality

\footnotetext{
${ }^{2} \mathrm{~A}$ codec is a device or computer program which can encode or decode a digital data stream or signal. In this thesis, the G729 codec, an ITU specified codec generally used in VOIP systems is considered.
} 
are directly proportional. If the $R$-factor is 100 , the voice quality is said to be the best. In this thesis, we consider $R$-factor after considering the subdivided impairment values of $R_{o}$, $I_{s}$ and $I_{d}$ in equation (3.4) is given by [13]

$$
R^{f a c}=R_{a}-\alpha_{1} \delta_{v}-\alpha_{2}\left(\delta_{v}-\alpha_{3}\right) H-\beta_{1}-\beta_{2} \log \left(1+\beta_{3} \psi\right)
$$

where $\psi$ is the packet loss percentage for the call and the other parameters $R_{a}, \alpha_{1}, \alpha_{2}, \alpha_{3}$, $\beta_{1}, \beta_{2}$ and $\beta_{3}$ are constants. The permitted values and range of values for these constants are specified in [26] from the E-model. In this thesis, values specified in [13] are considered. $R_{a}=94.2, \alpha_{1}=0.024, \alpha_{2}=0.11, \alpha_{3}=177.3, H=0$, if $\delta_{v}<\alpha_{3}$ otherwise $H=1$. $\beta_{1}, \beta_{2}$ and $\beta_{3}$ are codec dependent parameters. For G729 codec, these parameters are $\beta_{1}=$ $12, \beta_{2}=15, \beta_{3}=0.6$. The packet loss percentage can be substituted in terms of $\rho_{v}$ as $\psi=100 p_{v}=100\left(1-\rho_{v}\right)$. Then the utility function for voice in terms of $\delta_{v}$ and $1-\rho_{v}$ is

$$
U_{v}\left(\rho_{v}, \delta_{v}\right)=R_{a}-\alpha_{1} \delta_{v}-\alpha_{2}\left(\delta_{v}-\alpha_{3}\right) H-\beta_{1}-\beta_{2} \log \left(1+100 \beta_{3}\left(1-\rho_{v}\right)\right) .
$$

The average packet delay, $\delta_{v}$ is given by $[2,39,13]$

$$
\delta_{v}=\frac{K}{2}\left(\frac{1}{R}+\frac{1}{R-R_{v}}\right)
$$

The $R$-factor increases as $\delta_{v}$ decreases. Substituting $\delta_{v}$ in equation (3.6) we get

$$
\begin{aligned}
U_{v}\left(R_{v}, \rho_{v}\right)= & R_{a}-\alpha_{1} \frac{K}{2}\left(\frac{1}{R}+\frac{1}{R-R_{v}}\right)-\alpha_{2}\left(\frac{K}{2}\left(\frac{1}{R}+\frac{1}{R-R_{v}}\right)-\alpha_{3}\right) H- \\
& \beta_{1}-\beta_{2} \log \left(1+100 \beta_{3}\left(1-\rho_{v}\right)\right) .
\end{aligned}
$$

\subsection{Maximizing Total Utility using Lagrange Multipli- ers Method}

The rate optimization problem for the network is solved by maximizing the sum of utilities of all voice and data users. It can be formulated as

$$
\operatorname{maximize} U=U_{d}\left(R_{d}, \rho_{d}, p_{d}\right)+U_{v}\left(R_{v}, \rho_{v}\right)
$$

subject to $R_{d}+R_{v}=R$ 
In general, if $f(x, y)$ is an equation to be maximized with respect to the constraint $g(x)=c$ then the Lagrange function is given by [40]

$$
\Lambda(x, y, \lambda)=f(x, y)-\lambda(g(x, y)-c)
$$

The rate optimization problem in (3.9) can be written in the form of (3.10) by setting $x=R_{d}$, $y=R_{v}$ and $c=R_{d}+R_{v}-R$ and is given by

$$
\Lambda\left(R_{d}, R_{v}, \lambda\right)=U_{d}\left(R_{d}, \rho_{d}, \delta_{d}\right)+U_{v}\left(R_{v}, \rho_{v}\right)-\lambda\left(R_{d}+R_{v}-R\right)
$$

Solving the equation (3.11) using Lagrange multipliers method and KKT conditions gives the optimal values of $R_{d}$ and $R_{v}$.

Theorem 3.1 The optimal rate allocated to data traffic is the value of $R_{d}$ that satisfies the equation

$$
\frac{w_{d}\left[\rho_{d}\right]}{a}-\frac{1-w_{d}}{b} \times \frac{K}{2}\left[\frac{a}{\left(R-R_{d}\right)^{2}}\right]+\frac{K \alpha_{1}}{2} \times \frac{1}{R_{d}^{2}}+\frac{\alpha_{2} H K}{2 R_{d}^{2}}=0 .
$$

Proof 3.1 Refer Appendix A for the proof.

In this thesis, rate allocation is done to achieve maximum utility in the whole network. In order to achieve fairness for data and voice users, we can add minimum achievable utility constraints for voice and data traffic while solving optimization problem. By adding this, minimum required utility can be guaranteed to both data and voice users separately.

\subsection{Numerical Results}

Figure 3.1 provide numerical results for the network described in the Section 3.1. We consider packet size $K=5$; packet loss, $p_{d}=10^{-6}$ and $p_{v}=10^{-3}$; data parameters $r_{d}^{\max }=R$, $r_{d}^{\min }=0.1, \delta_{d}^{\min }=0 \mathrm{sec} /$ wireless $\mathrm{Hz}, \delta_{d}^{\max }=0.2 \mathrm{sec} /$ wireless $\mathrm{Hz}$ and weight $w_{d}=0.7$. Total utility, the sum of utilities of voice and data is calculated for three different values of $R$ and rate allocation is done at the point where maximum total utility occurs as shown in the Figure 3.1. When sufficient amount of rate is allocated to data and voice, total utility almost becomes constant and maximum total utility is achieved at the point where delay for the traffic is minimum. 


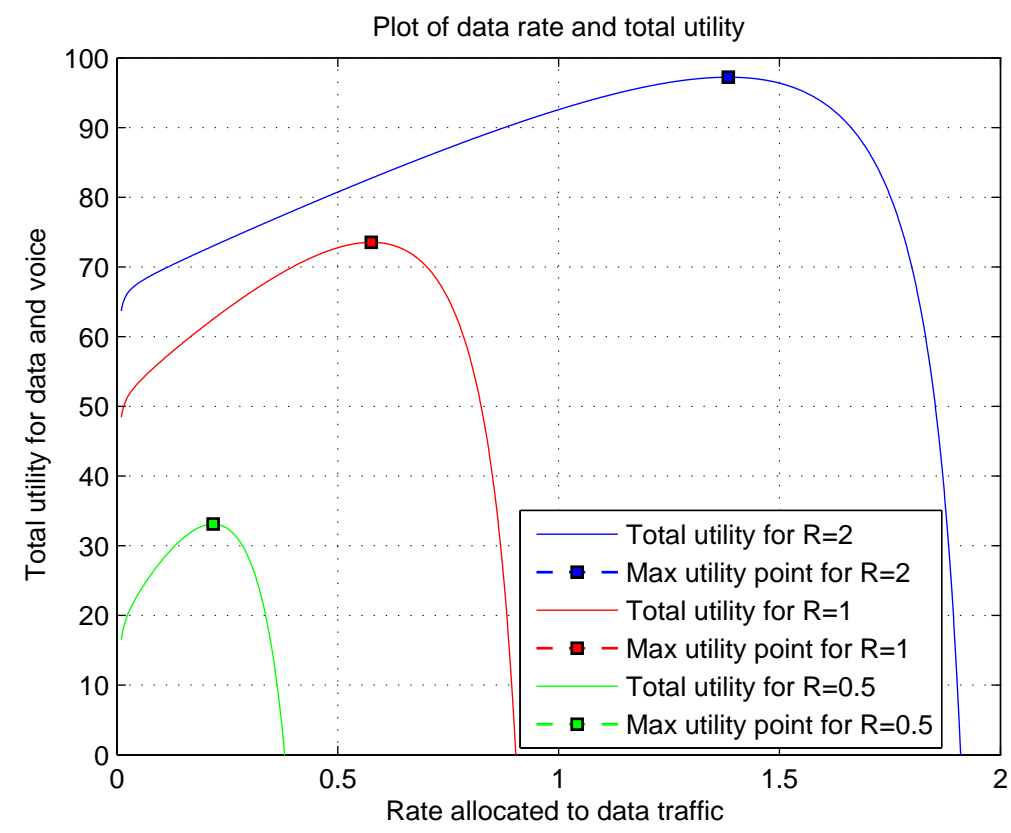

Figure 3.1: Total utility plot for a network with increase in rate allocated to data traffic

\subsection{Summary}

Traffic optimization problem is solved by maximizing the sum of utilities of all voice and data users in the network and optimal rates for data and voice are calculated. Optimization problem is solved using Lagrange multipliers method and KKT conditions. Optimal rate allocation is done where sufficient rate is allocated to data and voice. 


\section{Chapter 4}

\section{Power Allocation in a Point to Point Wireless Network with an Additional Mode}

Generally, wireless networks consist of nodes that communicate over a common wireless channel. As discussed previously, when number of nodes increases in a fixed area, communicating ability between the nodes becomes poor. In [23], it is shown that the throughput furnished to each user diminishes to zero as the number of users increases in a fixed area. As discussed in Section 2.5, the throughput problem cannot be alleviated by any combination of coding, modulation, or networking protocol [24]. So, we can add an additional mode of communication like wire in a wireless network to reduce the total load on wireless channel.

In multimodal networks, the nodes in addition to wireless transceivers have ability to send or receive wired transmission from other nodes. Here wired and/or wireless modes operate simultaneously to improve the performance of the wireless network. Multimodal networks can be used in some scenarios like cellular systems and wire-infrastructure based ad hoc networks, where base stations are connected by wires and close enough to exchange some wireless energy. They can also be used in electrical power system networks, which currently make use of wireless nodes on poles physically co-located with power lines that can also be used for communication. In our lab, we are currently building prototypes of wireless

\footnotetext{
${ }^{1}$ Part of the work presented in this chapter appears in [37].
} 
sensor networks using ultrasonic and NFMR links.

In Section 2.5, we have seen that power allocation is a crucial part for multimodal networks. Even if we connect the nodes that use wires to a power source, power allocation must be done to both modes as nodes connected to a power source will have infinite energy but not infinite power. In this chapter, power allocation for a source-destination pair multimodal network is done by analyzing outage probability and instantaneous rate plots.

\subsection{Network Model}

We consider a source-destination pair as shown in Figure 4.1. Information is transmitted from the source $S$ to the destination $D$ through two links which are wired (non-fading AWGN) and wireless channels that operate simultaneously. The wireless channel is attenuated by a fading coefficient $h_{w l}$ which undergoes Rayleigh fading and $h_{w}$ is the channel gain in wired channel. The received signal is also assumed to be corrupted by white Gaussian noise. In this thesis, the power allocation problem for the network shown in Figure 4.1 is

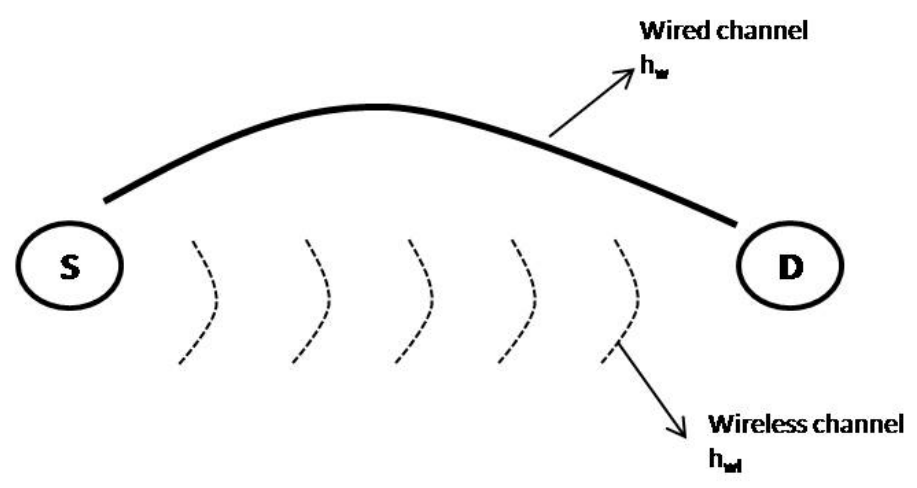

Figure 4.1: Wireless network with additional mode of communication (wire)

studied in two cases:

1. To achieve maximum instantaneous rate in the network

2. To achieve minimum outage probability in the network 
Let $P$ be the total transmit power. Then power $P_{w}$ is allocated to the wired channel and $P_{w l}$ to the wireless channel such that $P_{w}+P_{w l}=P$ in those two cases.

\subsection{Instantaneous Rate Maximization}

The information capacity for the wired channel from the equation (2.15) is given by

$$
\begin{aligned}
C_{w} & =B_{w} \log _{2}\left(1+\left|h_{w}\right|^{2} \mathrm{SNR}_{w}\right) \mathrm{bits} / \mathrm{sec} \\
& =B_{w} \log _{2}\left(1+\frac{\left|h_{w}\right|^{2} P_{w}}{N_{w} B_{w}}\right) \mathrm{bits} / \mathrm{sec}
\end{aligned}
$$

where $B_{w}$ is the bandwidth, $h_{w}$ is the fading coefficient, $N_{w}$ is the noise spectral density of the wired channel, $\mathrm{SNR}_{w}=\frac{P_{w}}{N_{w} B_{w}}$ is the signal to noise ratio of wired channel and $h_{w}$ is considered to be constant for the wired channel.

The instantaneous capacity for the wireless channel is given by

$$
\begin{aligned}
C_{w l} & =B_{w l} \log _{2}\left(1+\left|h_{w l}\right|^{2} \mathrm{SNR}_{w l}\right) \mathrm{bits} / \mathrm{sec} \\
& =B_{w l} \log _{2}\left(1+\frac{\left|h_{w l}\right|^{2} P_{w l}}{N_{w l} B_{w l}}\right) \mathrm{bits} / \mathrm{sec}
\end{aligned}
$$

where $B_{w l}$ is the bandwidth, $h_{w l}$ is the fading coefficient, $N_{w l}$ is the noise spectral density and $\mathrm{SNR}_{w l}=\frac{P_{w l}}{N_{w l} B_{w l}}$ is the signal to noise ratio of the wireless channel. The fading coefficient $h_{w l}$ varies for the wireless channel.

The total instantaneous capacity for the multimodal network is the sum of channel capacity of wired channel and instantaneous capacity of the wireless channel. Adding equations (4.1) and (4.2) yields, [41]

$$
\begin{aligned}
C & =C_{w}+C_{w l} \\
& =B_{w} \log _{2}\left(1+\left|h_{w}\right|^{2} \mathrm{SNR}_{w}\right)+B_{w l} \log _{2}\left(1+\left|h_{w l}\right|^{2} \mathrm{SNR}_{w l}\right) \mathrm{bits} / \mathrm{sec} \\
& =\frac{B_{w}}{B_{w l}} \log _{2}\left(1+\left|h_{w}\right|^{2} \mathrm{SNR}_{w}\right)+\log _{2}\left(1+\left|h_{w l}\right|^{2} \mathrm{SNR}_{w l}\right) \mathrm{bits} / \mathrm{sec} / \text { wireless Hz } \\
& =\frac{B_{w}}{B_{w l}} \log _{2}\left(1+\frac{\left|h_{w}\right|^{2} P_{w}}{N_{w} B_{w}}\right)+\log _{2}\left(1+\frac{\left|h_{w l}\right|^{2} P_{w l}}{N_{w l} B_{w l}}\right) \mathrm{bits} / \mathrm{sec} / \text { wireless Hz. }
\end{aligned}
$$

To achieve maximum rate in the network, the total rate in (4.3) must be maximum. So, the 
rate optimization problem can be formulated as

$$
\begin{aligned}
\operatorname{maximize} C & =C_{w}+C_{w l} \\
\text { subject to } P_{w}+P_{w l} & =P .
\end{aligned}
$$

The Lagrange equation for the rate optimization problem can be written as

$$
\Lambda\left(P_{w}, P_{w l}, \lambda\right)=\frac{B_{w}}{B_{w l}} \log _{2}\left(1+\frac{\left|h_{w}\right|^{2} P_{w}}{N_{w} B_{w}}\right)+\log _{2}\left(1+\frac{\left|h_{w l}\right|^{2} P_{w l}}{N_{w l} B_{w l}}\right)+\lambda\left(P_{w}+P_{w l}-P\right) .
$$

Solving the equation (4.4) using Langrange multipliers method and applying KKT conditions gives the optimal values of $R_{d}$ and $R_{v}$.

Theorem 4.1 Optimal values of $P_{w}$ and $P_{w l}$ to achieve maximum instantaneous rate in source-destination multimodal network are

$$
\begin{aligned}
P_{w} & =\frac{B_{w} B_{w l} N_{w l}\left|h_{w}\right|^{2}+B_{w}\left|h_{w}\right|^{2}\left|h_{w l}\right|^{2} P-\left|h_{w l}\right|^{2} B_{w} B_{w l} N_{w}}{\left|h_{w}\right|^{2}\left|h_{w l}\right|^{2}\left(B_{w}+B_{w l}\right)} \\
P_{w l} & =P-P_{w} .
\end{aligned}
$$

Proof 4.1 Refer Appendix B for the proof.

\subsection{Outage Probability Minimization}

The outage probability for a fading channel from (2.22) is given by

$$
P_{\text {out }}(R) \approx \frac{2^{R}-1}{\mathrm{SNR}} \text { at high SNR. }
$$

Now, outage probability for the multimodal network shown in Figure 4.1 is given by [41]

$$
\begin{aligned}
P_{\text {out }}\left(R, \mathrm{SNR}_{w l}\right) & =\operatorname{Prob}(C<R) \\
& =\operatorname{Prob}\left(C_{w l}<R-C_{w}\right) \\
& =1-e^{-\left(\frac{2^{R-C_{w}-1}}{\mathrm{SNR}_{w l}}\right)} \\
& \approx \frac{2^{R-C_{w}}-1}{\mathrm{SNR}_{w l}} \text { at high SNR } \\
& \approx \frac{2^{R-B_{w} \log _{2}\left(1+\frac{\left|h_{w}\right|^{2} P_{w}}{N_{w} B_{w}}\right)}-1}{\frac{P_{w l}}{N_{w l} B_{w l}}} .
\end{aligned}
$$


The equation (4.9) is to be minimized w.r.t constraint $P_{w}+P_{w l}=P$. Outage probability optimization problem is formulated as

$$
\begin{aligned}
\operatorname{minimize} P_{\text {out }}(R, \mathrm{SNR}) & =\frac{2^{R-B_{w} \log _{2}\left(1+\frac{\left|h_{w}\right|^{2} P_{w}}{N_{w} B_{w}}\right)}-1}{\frac{P_{w l}}{N_{w l} B_{w l}}} \\
\text { subject to } P_{w}+P_{w l} & =P .
\end{aligned}
$$

The Lagrange equation for the outage minimization problem can be written as

$$
\Lambda\left(P_{w}, P_{w l}, \lambda\right)=\frac{2^{R-B_{w} \log _{2}\left(1+\frac{\left|h_{w}\right|^{2} P_{w}}{N_{w} B_{w}}\right)}-1}{\frac{P_{w l}}{N_{w l} B_{w l}}}+\lambda\left(P_{w}+P_{w l}-P\right) .
$$

Minimizing the equation (4.12) using Langrange multipliers method gives the optimal values of $P_{w}$ and $P_{w l}$.

Theorem 4.2 Optimal values of $P_{w}$ and $P_{w l}$ are the values that satisfies the simultaneous equations

$$
\begin{gathered}
\left(2^{R-\left(B_{w} / B_{w l}\right) \log \left(1+\frac{\left|h_{w}\right|^{2} P_{w}}{N_{w} B_{w}}\right)}\right)\left(\frac{N_{w l}}{P_{w l}}\right) \frac{-\left|h_{w}\right|^{2} B_{w}}{\left(N_{w} B_{w}+\left|h_{w}\right|^{2} P_{w}\right)}+\lambda_{1}=0 \text { and } \\
\left(2^{R-\left(B_{w} / B_{w l}\right) \log \left(1+\frac{\left|h_{w}\right|^{2} P_{w}}{B_{w} N_{w}}\right)}-1\right)\left(N_{w l} B_{w l} \frac{-1}{P_{w l}^{2}}\right)+\lambda_{1}=0 .
\end{gathered}
$$

Proof 4.2 Refer Appendix C for the proof.

\subsection{Numerical Results}

Figure 4.2 shows power optimization problem results for the maximum instantaneous capacity scenario for the network shown in the Figure 4.1. Outage probability vs wired power plots are drawn for different bandwidth ratios of wired and wireless channels. Pathloss and fading effects are considered and pathloss exponent value of 3 is used for calculations. Total instantaneous rate and optimal wired power are more when $B_{w}=10 \times B_{w l}$. When channel bandwidths are equal, optimal wired power and wireless power are almost equal. When $B_{w}=\frac{1}{10} B_{w l}$, total instantaneous capacity is less compared to former cases and optimal wireless power is more than optimal wired power. As capacity depends on the value of $h$, 


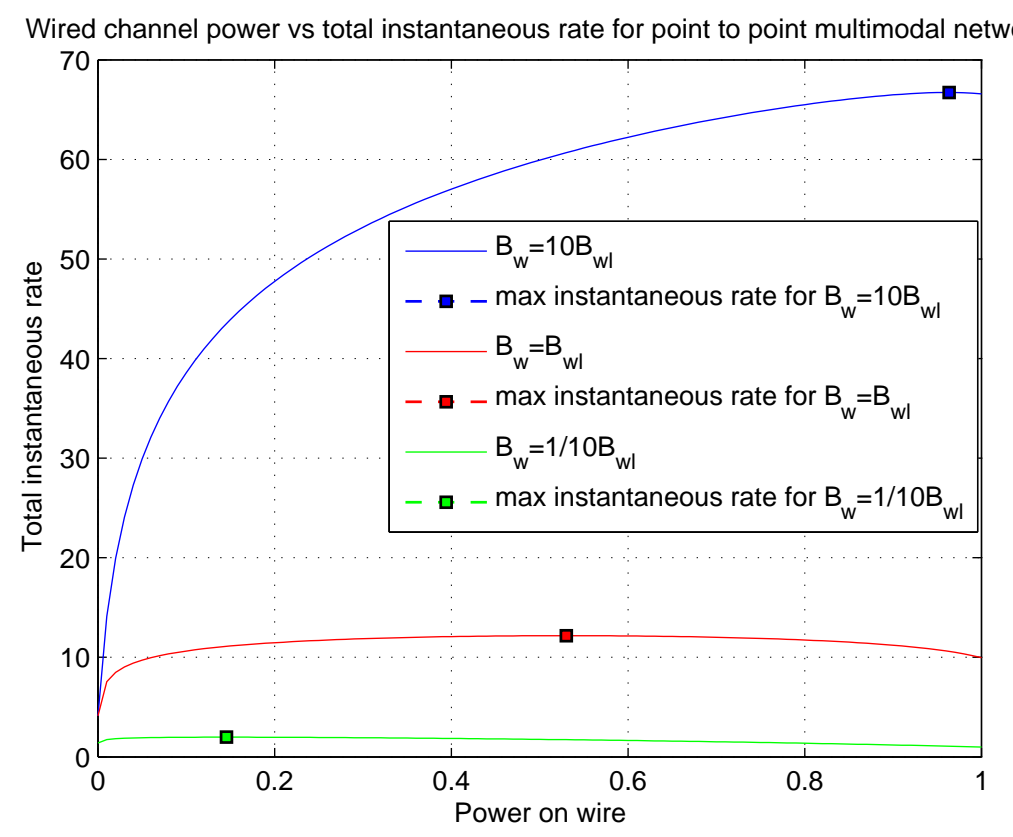

Figure 4.2: Plot of wired power vs total instantaneous rate for a multimodal network for various bandwidth ratios of wired and wireless channel

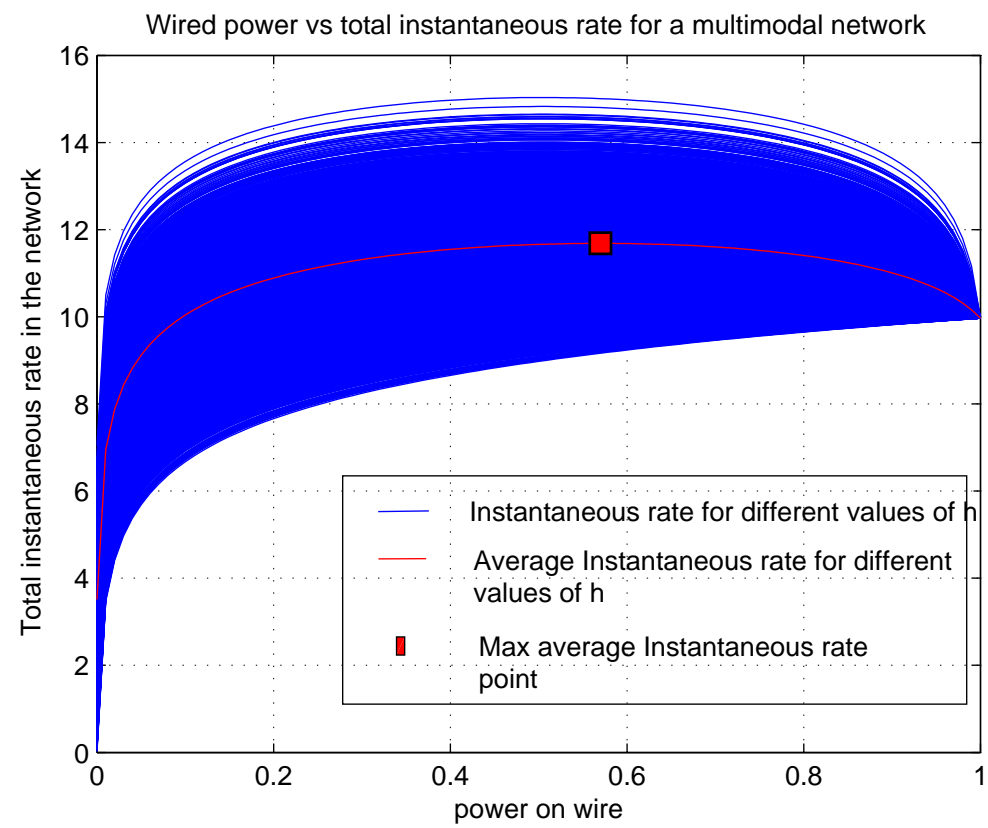

Figure 4.3: Plot of wired power vs total instantaneous rate for a multimodal network for different values of wireless gain $\mathrm{h}$

in Figure 4.3, the total instantaneous rate and average of instantaneous rate vs wired power are plotted for different values of $h$. The maximum average instantaneous rate point for 
$B_{w}=B_{w l}$ case is also plotted in the Figure 4.3.

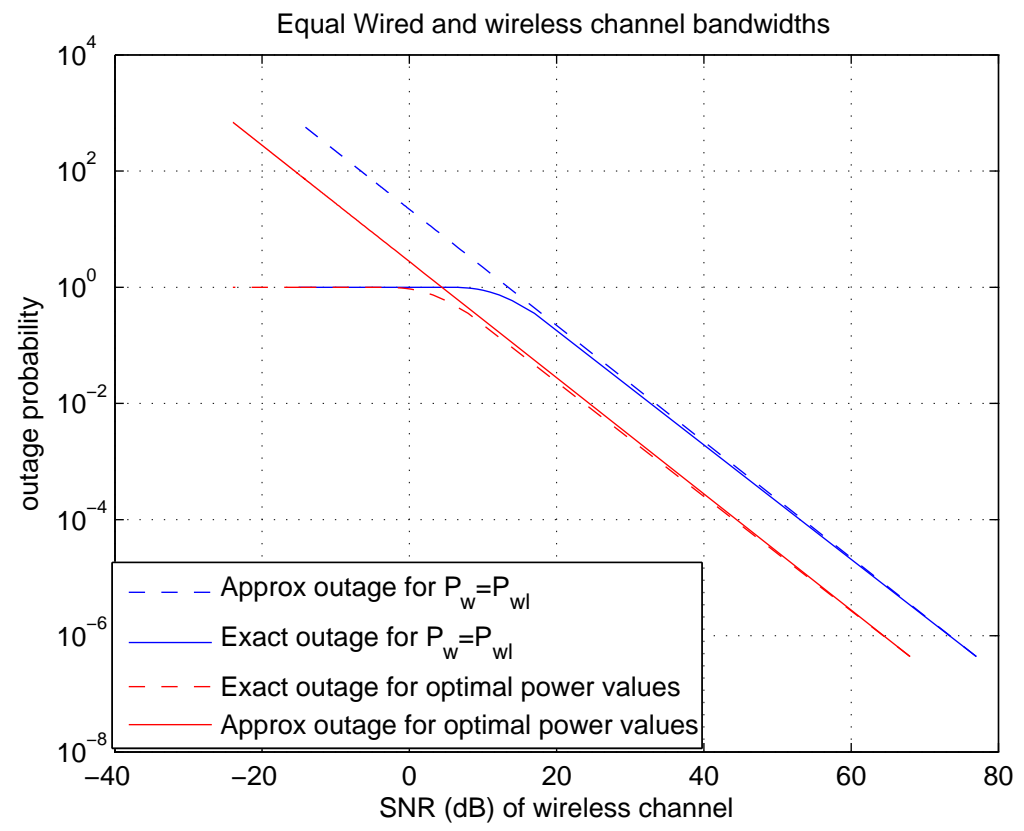

Figure 4.4: Plot of wired power vs outage probability for a multimodal network for $B_{w}=B_{w l}$

Figures 4.4, 4.5 and 4.6 show the results for power optimization problem to achieve minimum outage probability for the network shown in the Figure 4.1 for different ratios of wired and wireless channel bandwidths. Exact and approximate values of outage probability for different ratios of wired and wireless channel bandwidths are plotted using equations (4.8) and (4.9) respectively. Outage probability with optimal power values is plotted by varying the $\mathrm{SNR}_{w l}$. From the plots, it is observed that the outage probability with optimal power values is less than the outage with equal power values. More gain in outage probability is observed when $B_{w}=10 \times B_{w l}$ which can be seen in Figure 4.6. 


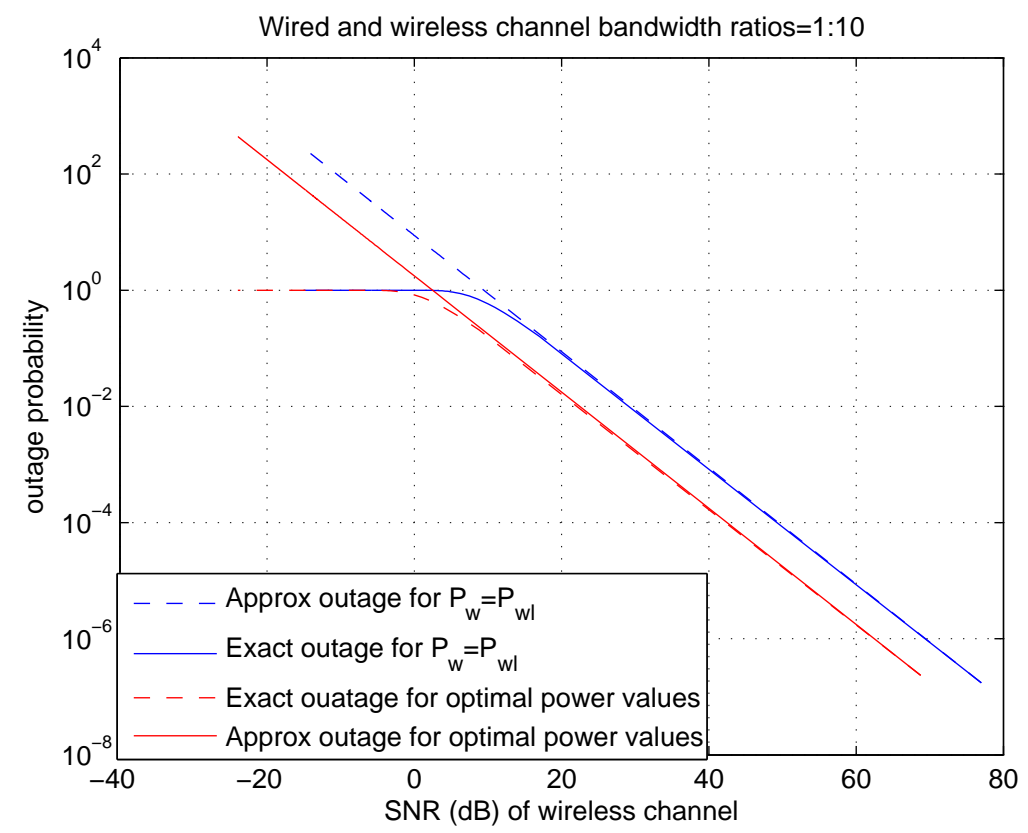

Figure 4.5: Plot of wired power vs outage probability for multimodal network for $B_{w}=\frac{1}{10} B_{w l}$

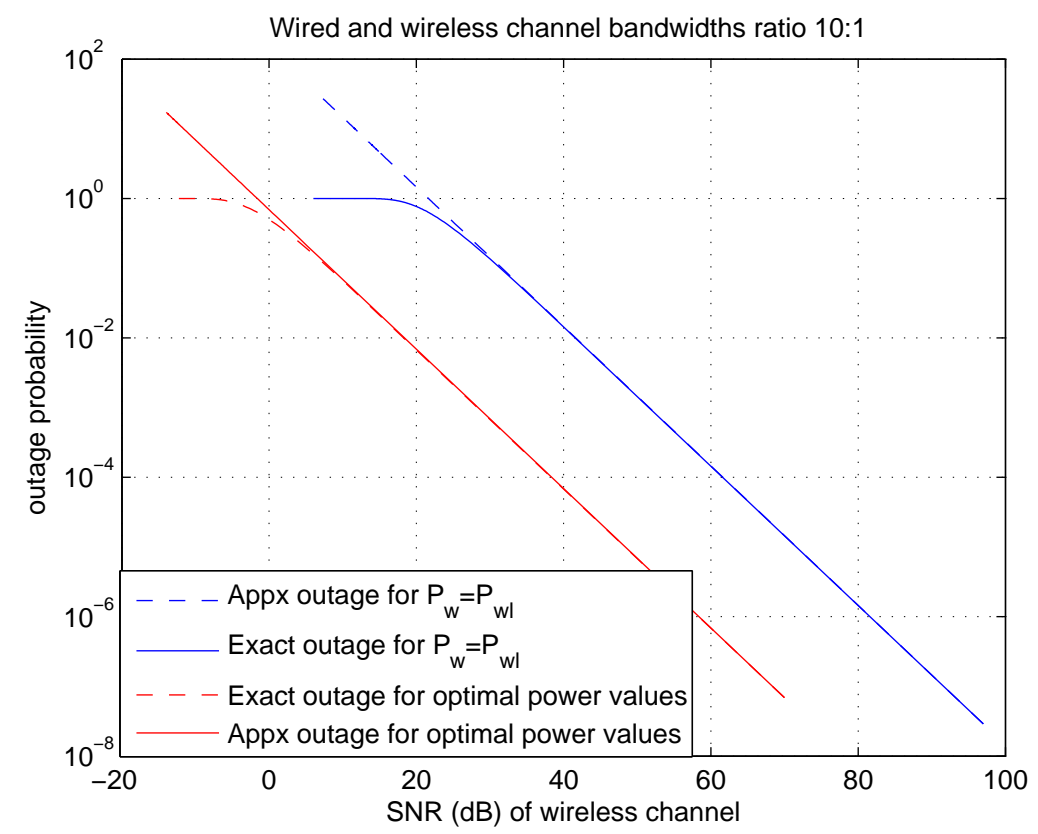

Figure 4.6: Plot of wired power vs outage probability for multimodal network for $B_{w}=10 B_{w l}$

\subsection{Summary}

A source-destination pair multimodal network is analyzed through instantaneous rate and high-SNR outage probability expressions. Optimal power values of wired and wireless 
channel are calculated maximizing instantaneous rate and minimizing outage probability. Instantaneous rate and outage probability are calculated for different bandwidth ratios of wired and wireless channel. Optimization problems are solved using Lagrange multipliers and KKT conditions. 


\section{Chapter 5}

\section{Outage Probability Minimization in a Cooperative Multimodal Network}

In Chapter 2, we have studied that the cooperative diversity techniques increase the performance of a wireless channel. In chapter 4, we have optimized instantaneous rate and outage probability for a source-destination pair multimodal network. In [21], the authors analyzed repetition-based and space-time coded cooperative diversity for cooperative wireless relay networks using outage probability as a performance measure. The network and protocol models used by them are studied in Section 2.4. In this chapter, a diamond multimodal cooperative network, where relays have capability to communicate via wired and wireless channels as previously discussed in Section 2.5 is considered and optimal power allocation is done analytically using high-SNR outage probability expressions from [25]. The outage probability for the network is considered in two different network topologies.

- Wire placed between source and relay

- Wire placed between relay and destination

In this part of the thesis, some results from [25] are duplicated and analyzed. So the network and outage probability expressions from [25] are considered in this chapter.

\footnotetext{
${ }^{1}$ Part of the work presented in this chapter appears in [37]
} 


\section{$5.1 \quad$ Network Model}

In this thesis, we consider a cooperative diamond relay network as shown in Figure 5.1. The source $s$ broadcasts the information to the destination $d(s)$. Two relays $r_{1}$ and $r_{2}$ account to the cooperating terminals together with the destination $d(s) . r_{w}$ is used for the relay where wire is attached. $\lambda$ is the Rayleigh fading coefficient for each channel. Channel gains $a_{s, r_{n}}, a_{s, d(s)}$ are between source and relay $r_{n}$ and source and destination, $a_{r_{n}, d(s)}$ between relays and destination. $1 / \lambda_{s, r_{n}}, 1 / \lambda_{s, d(s)}, 1 / \lambda_{r_{n}, d(s)}$ are variances for corresponding channel gains. Channel gains are considered to be constant during the transmission. As decode and forward cooperative diversity protocol is considered, the decoding set $D(s)^{1}$ can be \{\}$,\left\{r_{1}\right\},\left\{r_{2}\right\}$ or $\left\{r_{1}, r_{2}\right\}$.

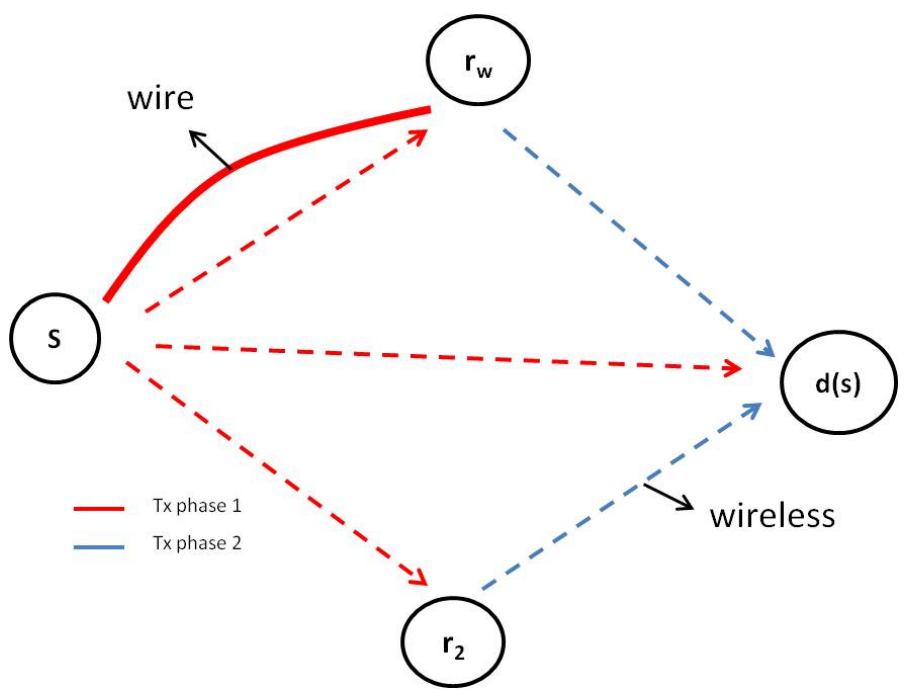

Figure 5.1: Cooperative relay network with wire between source and relay

Figures 5.1 and 5.2 describe the network topologies with wire placed between sourcerelay and relay-destination respectively. Assume additional mode (wire) has bandwidth $B_{w}$. Channel gain $h$ is also assumed to be constant across the wired channel. Let $B_{w l}$ be the bandwidth of the wireless channel. In space-time coded cooperative diversity, the terminals transmit in half the available degrees of freedom, so the SNR in the network is normalized

\footnotetext{
${ }^{1}$ The decoding set $D(s)$ is the set of relays that can decode the message after every transmission.
} 


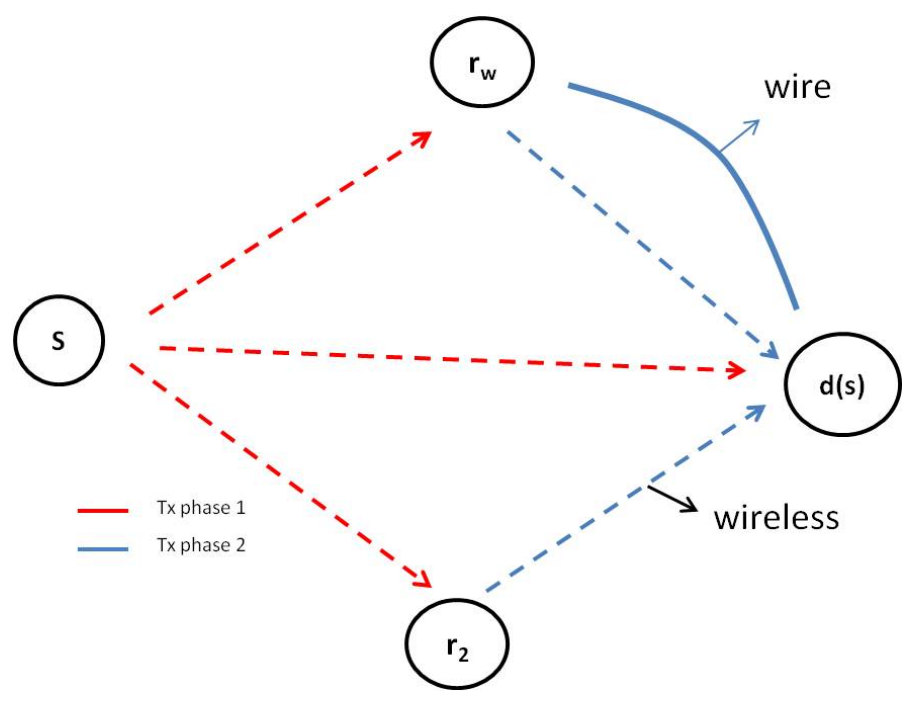

Figure 5.2: Cooperative relay network with wire between relay and destination

$\frac{2}{m}$ SNR. Power is divided between two channels. $P_{w}$ is the percentage of power assigned to the additional mode which varies between 0 and 1 . As wire is attached to relay $r_{1}, r_{1}=r_{w}$ is used.

Let $I_{s t c}<R$ be an outage event that the mutual information of the network $I_{s t c}$ falls below some fixed spectral efficiency $R$. Then the probability $P_{r}\left[I_{s t c}<R\right]$ is considered as the outage probability of the network. Since $D(s)$ is also a random set, to calculate outage probability, we will use the total probability law [21]

$$
P_{r}\left[I_{s t c}<R\right]=\sum_{D(s)} P_{r} D(s) P_{r}\left[I_{\text {out }}<R \mid D(s)\right]
$$

\subsection{Outage Probability for the Network with Wire be- tween Source and Relay}

In this section, outage probability for the first network topology as shown in the Figure 5.1 is studied. Mutual information for the network is the sum of mutual information of two parallel channels $[25,21]$

$$
I=\frac{1}{2} \log \left(1+\frac{2\left(1-p_{w}\right)}{m} \mathrm{SNR}\left|a_{s, r_{w}}\right|^{2}\right)+\frac{1}{2} \frac{B_{w}}{B_{w l}} \log \left(1+\frac{2 P_{w}}{m B_{w} / B_{w l}} \mathrm{SNR}|h|^{2}\right)
$$


the units are normalized to the bandwidth of the wireless channel $B_{w l}$. The total probability (5.1) is divided into two cases where $r_{w} \in D(s)$ and $r_{w} \notin D(s)$. Then outage probability becomes [25]

$$
P_{r}[I<R]=\sum_{D(s): r_{w} \in D(s)} P_{r}[D(s)] P_{r}\left[P_{r}<R \mid D(s)\right]+\sum_{D(s): r_{w} \notin D(s)} P_{r}[D(s)] P_{r}\left[P_{r}<R \mid D(s)\right] .
$$

As $D(s)$ has nothing to do much in the transmission of the signal from source to destination, the conditional probability does not change much with the addition of non-fading AWGN channel. Let $\lambda_{s, r_{\text {orig }}}=\lambda_{s, r}$. For wired relay, redefining $\lambda_{s, r}=\frac{\lambda_{s, r_{\text {orig }}}\left(2^{2 \hat{R}}-1\right)}{2^{2 R}-1}$ where $\hat{R}=$ $R-\frac{1}{2} \frac{B_{w}}{B_{w l}} \log \left(1+\frac{2 P_{w}}{\left(m B_{w} / B_{w l}\right) \operatorname{SNR}|h|^{2}}\right)$. Now, outage probability is similar to the expression (2.34). At high-SNR, the information outage probability is given as [25]

$$
\begin{aligned}
P_{r}[I<R] \approx & {\left[\frac{2^{2 R}-1}{2 \mathrm{SNR} / m}\right] \times \sum_{D(s)}\left[\frac{1}{1-P_{w}}\right]^{m-|D(s)|-1} } \\
& \times \lambda_{s, d(s)} \prod_{r \notin D(s)} \lambda_{s, r} \prod_{r \in D(s)} \lambda_{r, d(s)} \times A_{D(s)}\left(2^{2 R}-1\right)
\end{aligned}
$$

where $A_{n}(t)=\frac{1}{(n-1) !} \int_{0}^{1} \frac{w^{(n-1)}(1-w)}{(1+t w)} \mathrm{d} w, \ldots n>0$ and $A_{o}=1$.

The expression for the information outage probability for the network with wire between source-relay for the network shown in Figure 5.1 from the equation (5.4) is given by [25]

$$
\begin{aligned}
P_{r}[I<R] \approx & \left(\frac{2^{2 R}-1}{2 \mathrm{SNR} / m}\right) \times \lambda_{s, d(s)}\left[\left(\frac{1}{1-P_{w}}\right)^{2} \times \lambda_{s, r_{w}}\left(2^{2 R-\frac{B_{w}}{B_{w l}} \times \log \left(1+\frac{2 P_{w}}{m B_{w} / B_{w l}} \mathrm{SNR}|h|^{2}\right)}\right) \times \lambda_{s, r_{1}}\right. \\
& +\left(\frac{1}{1-P_{w}}\right) \times \lambda_{s, r_{w}} \times\left(2^{2 R-\frac{B w}{B_{w l}} \log \left(1+\frac{2 P_{w}}{m B_{w} / B_{w l}} \mathrm{SNR}|h|^{2}\right)}\right) \times \lambda_{r_{1}, d(s)} \times A_{1}\left(2^{2 R}-1\right) \\
& +\left(\frac{1}{1-P_{w}}\right) \times \lambda_{r_{w}, d(s)} \times \lambda_{s, r_{1}} \times A_{1}\left(2^{2 R}-1\right)+\lambda_{r_{w}, d(s)} \\
& \left.\times \lambda_{r_{1}, d(s)} \times A_{2}\left(2^{2 R}-1\right)\right] .
\end{aligned}
$$

Theorem 5.1 The optimal power for the wired channel is the value of $P_{w}$ that satisfies the equation

$$
\begin{aligned}
& \left.\left[\lambda_{s, r_{w}} \times\left(2^{2 R-\frac{B_{w}}{B_{w l}} \log \left(1+\frac{2 P_{w}}{m \frac{B w}{B}} \mathrm{SNR}|h|^{2}\right.}\right)\right) \times\left(\frac{2 \lambda_{s, r_{1}}}{\left(1-P_{w}\right)^{3}}+\frac{1}{\left(1-P_{w}\right)^{2}} \lambda_{r_{1}, d(s)} A_{1}\left(2^{2 R}-1\right)\right)\right] \\
& +\left[\left(\frac{\lambda_{s, r_{1}}}{\left(1-P_{w}\right)^{2}}+\frac{1}{\left(1-P_{w}\right)} \lambda_{r_{1}, d(s)} A_{1}\left(2^{2 R}-1\right)\right) \times\left(\lambda_{s, r_{w}} \times\left(2^{2 R-\frac{B_{w}}{B_{w l}} \log \left(1+\frac{2 P_{w}}{m \frac{B_{w}}{B_{w l}}} \mathrm{SNR}|h|^{2}\right.}\right)\right)\left(\frac{\frac{-2}{m} \mathrm{SNR}|h|^{2}}{1+\frac{2 P_{w}}{m \frac{B w}{B}} \operatorname{SNR}|h|^{2}}\right)\right] \\
& +\frac{1}{\left(1-P_{w}\right)^{2}} \lambda_{r_{w} d(s)} \lambda_{s, r_{1}} A_{1}\left(2^{2 R}-1\right)=0 .
\end{aligned}
$$


Proof 5.1 Refer Appendix D.1 for the Proof.

\subsection{Outage Probability for the Network with Wire be- tween Relay and Destination}

When wire is placed between $r_{w}$ and $d(s)$ as shown in Figure 5.2, if the relay decodes the message from the source, the mutual information between the transmitter and destination is

$$
\begin{aligned}
I= & \frac{1}{2} \log \left(1+\frac{2}{m} \operatorname{SNR}\left|a_{s, d(s)}\right|^{2}\right)+\frac{1}{2} \log \left(1+\frac{2}{m} \operatorname{SNR}\left[\left(1-P_{w}\right)\left|a_{r_{w}, d(s)}\right|^{2}\right.\right. \\
& \left.\left.+\sum_{r \in D(s): r \neq r_{w}}\left|a_{r, d(s)}\right|^{2}\right]\right)+\frac{1}{2} \frac{B_{w}}{B_{w l}} \log \left(1+\frac{2 P_{w}}{m B_{w} / B_{w l}} \mathrm{SNR}\right) .
\end{aligned}
$$

Splitting the equation (5.1) into cases where $r_{w} \in D(s)$ and $r_{w} \notin D(s)$, the total probability becomes

$$
P_{r}[I<R] \approx \sum_{D(s): r_{w} \in D(s)} P_{r}[D(s)] P_{r}\left[P_{r}<R \mid D(s)\right]+\sum_{D(s): r_{w} \notin D(s)} P_{r}[D(s)] P_{r}\left[P_{r}<R \mid D(s)\right]
$$

Outage probability expression for the network with wire between relay and destination is given by [25]

$$
\begin{aligned}
P_{r}(I<R) \approx & {\left.\left[\frac{1}{2 \mathrm{SNR} / m}\right]^{m} \times \lambda_{s, d(s)}\right) \times\left[\left(\frac{\lambda_{r_{w}, d(s)}}{\left(1-P_{w}\right)}\right.\right.} \\
& \times \sum_{D(s): r_{w} \in D(s)} \times\left(2^{2 \hat{R}}-1\right)^{|D(s)|+1} \times\left(2^{2 R}-1\right)^{m-|D(s)|-1} \\
& \left.\times \prod_{r \in D(s): r \neq r_{w}} \lambda_{r, d(s)} \prod_{r \notin D(s)} \lambda_{s, r} \times A_{|D(s)|}\left(2^{2 \hat{R}}-1\right)\right) \\
& \left.+\left(\left(2^{2 R}-1\right)^{m} \times \lambda_{s, r_{w}} \times \sum_{D(s): r_{w} \notin D(s)} \lambda_{s, r} \times A_{|D(s)|}\left(2^{2 R}-1\right)\right)\right] . \\
& \times \prod_{r \in D(s)} \lambda_{r, d(s)} \prod_{r \notin D(s): r \neq r_{w}}
\end{aligned}
$$

The outage probability expression for the network in Figure 5.2 with wire between relay and 
destination is $[25]$

$$
\begin{aligned}
P_{r}(I<R)= & \frac{1}{2 \mathrm{SNR} / m} \times \lambda_{r_{w}, d(s)} \times\left[\frac { \lambda _ { r _ { w } , d ( s ) } } { 1 - P _ { w } } \left(\left(2^{2 \tilde{R}}-1\right)^{2} \times\left(2^{2 R}-1\right) \times \lambda_{s, r_{2}} \times\right.\right. \\
& \left.A_{1}\left(2^{2 \tilde{R}}-1\right)+\left(2^{2 \tilde{R}}-1\right)^{3} \times \lambda_{r_{2}, d(s)} \times A_{2}\left(2^{2 \tilde{R}}-1\right)\right)+\left(\left(2^{2 R}-1\right)^{3} \times \lambda_{s, r_{w}}\right. \\
& \left.\left.\times\left(\lambda_{s, r_{2}}+\lambda_{r_{2}, d(s)} \times A_{1}\left(2^{R}-1\right)\right)\right)\right] .
\end{aligned}
$$

Theorem 5.2 The optimal power that can be allocated to wired channel is the $P_{w}$ value that satisfies the equation

$\left(2 t_{1} \times \frac{\mathrm{d} t_{1}}{\mathrm{~d} P_{w}} \times t \times \lambda_{s, r_{2}} \times f_{1}\right)+\left(t_{1}^{2} \times t \times \lambda_{s, r_{2}} \times \frac{\mathrm{d} f_{1}}{\mathrm{~d} P_{w}}\right)+\left(t_{1}^{2} \times t \times \lambda_{s, r_{2}} \times f_{1}\right)+\left(t_{1}^{3} \times \lambda_{r_{2}, d(s)} \times \frac{\mathrm{d} f_{2}}{\mathrm{~d} P_{w}}+3 t_{1}^{2}\right.$ $\left.\times \frac{\mathrm{d} t_{1}}{\mathrm{~d} P_{w}} \times \lambda_{r_{2}, d(s)} \times f_{2}\right)=0$

where $t=2^{2 R}-1, t_{1}=2^{2 \tilde{R}}-1, f=A_{1}\left(2^{2 R}-1\right), f_{1}=A_{1}\left(2^{\tilde{R}}-1\right), f_{2}=A_{2}\left(2^{2 \tilde{R}}-\right.$ 1) and $\frac{\mathrm{d}}{\mathrm{d} P_{w}}$ is the differential operator over $P_{w}$.

Proof 5.2 Refer Appendix D.2 for the Proof.

\subsection{Numerical Results}

Figures 5.3 and 5.4 show the outage probability curves and the minimum outage probability point found analytically using numerical analysis. The point is observed to be nearer to the minimum point from the outage probability plot. The outage probability is calculated numerically considering $m=3$ and the parameter $\lambda=10$ for the wireless channel corresponding to the additional mode and $\lambda=1$ for all other wireless channels which states that additional mode is placed where the wireless channel is bad. The optimal power allocation is done at the minimum outage probability point where the wired channel can support at least rate $R$. From the plots it is observed that when $P_{w}$ increases, the outage probability decreases as the power for other relay decreases reducing its decoding capability. Network performance is better when wire is placed between source-relay as outage probability for wire between source-relay case is less compared to relay-destination case. 


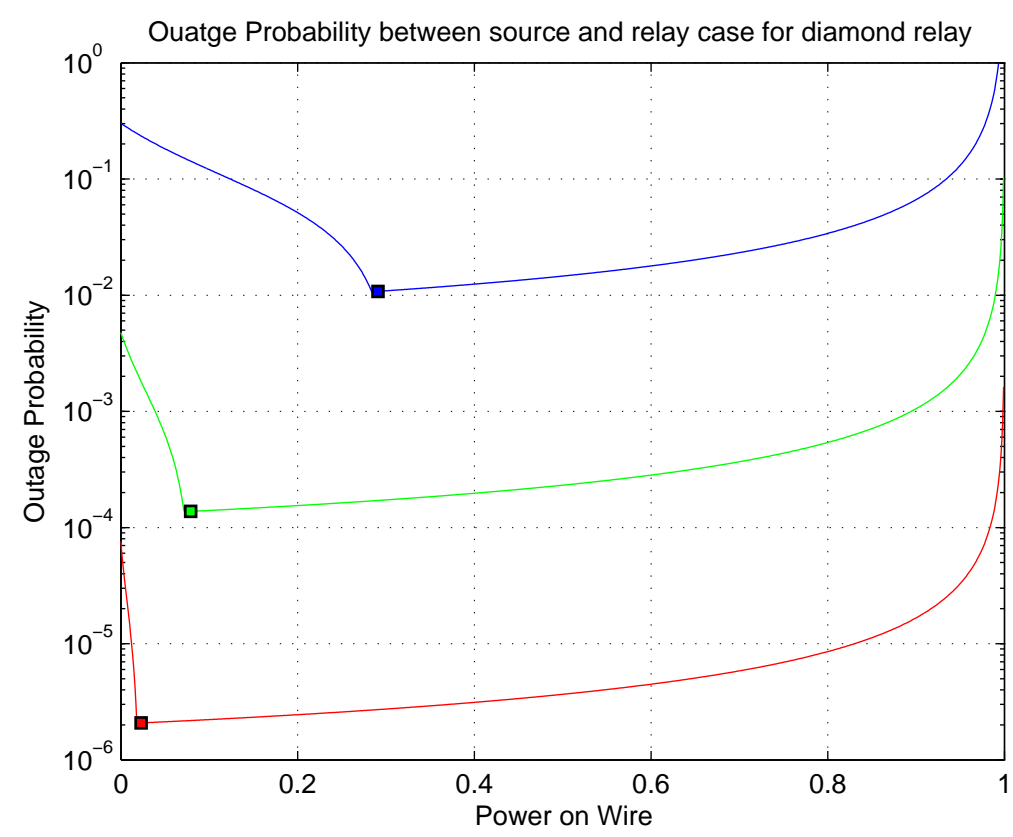

Figure 5.3: Outage probability plot for wire between source and relay for a diamond relay network with three power values, $12 \mathrm{~dB}$ (blue), 18dB (green), $24 \mathrm{~dB}$ (red) and the points for the corresponding plots are the optimal power points

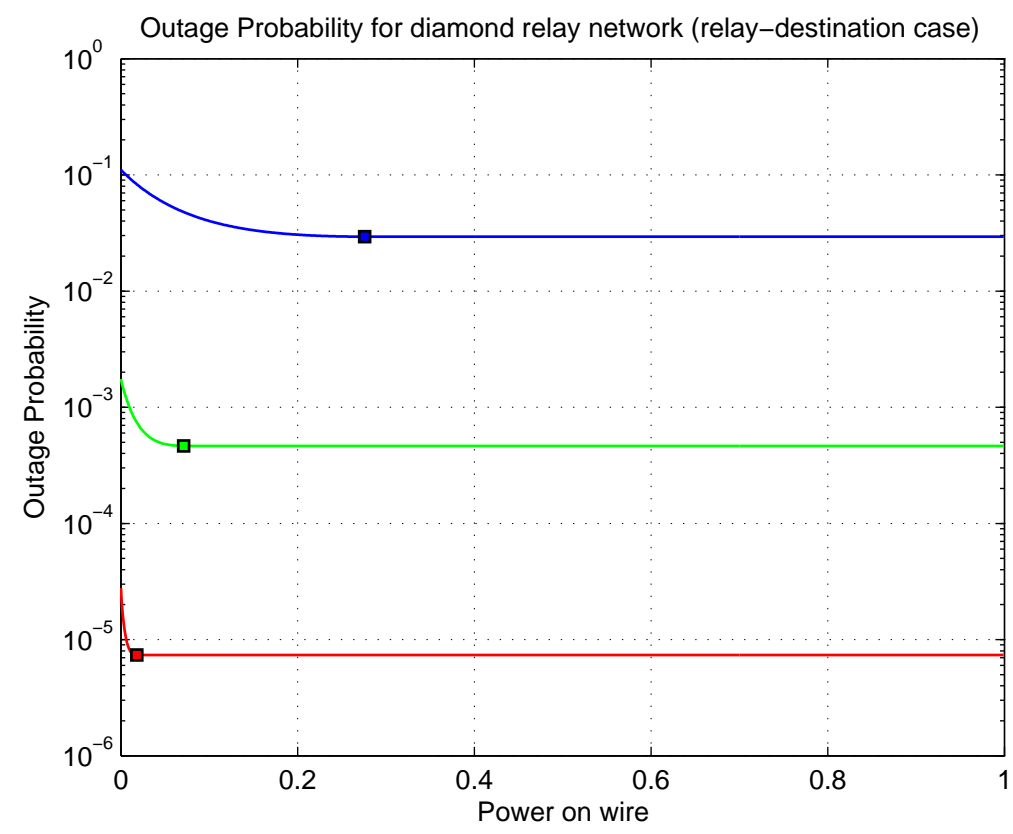

Figure 5.4: Outage probability plot for wire between relay and destination for a diamond relay network with three power values, $12 \mathrm{~dB}$ (blue), $18 \mathrm{~dB}$ (green), $24 \mathrm{~dB}$ (red) and the points for the corresponding plots are the optimal power points 


\subsection{Summary}

In this chapter, cooperative multimodal networks and their performance in terms of outage probability expressions are studied. Optimal power allocation is done for a space-time cooperative multimodal network by analytically minimizing outage probability expression from [25]. The minimum point calculated analytically is nearer to the minimum point from the outage probability plot. 


\section{Chapter 6}

\section{Conclusion and Future Work}

\subsection{Summary and Conclusions}

In this thesis, multimodal networks where other modes of communication like wires, infrared links operates in addition to wireless mode to improve the performance of wireless networks are studied. Rate and power allocation problems are considered for multimodal networks with delay-sensitive data and voice traffic. As utility is a better measure for analyzing performance of voice and data users, rate optimization is done to achieve maximum utility in the network. Utility function for data in terms of rate, reliability and delay; utility function for voice in terms of rate and delay are considered to calculate total utility. For power optimization, a source-destination pair network and a diamond cooperative network are considered. For analyzing the performance of the source-destination pair network, instantaneous rate and outage probability are studied.

Instantaneous rate maximization and outage probability minimization are proposed for optimal power allocation in source-destination pair multimodal networks. For cooperative multimodal networks, analytical minimization of outage probability is proposed for optimal power allocation to wired and wireless modes.

The performance of source-destination pair multimodal network is observed to be better when wired channel bandwidth is greater than wireless channel bandwidth. In such case, maximum instantaneous rate and minimum outage probability are observed after optimal power allocation. So, we can conclude that rate and outage probability optimizations improve 
the performance of the network. In a cooperative multimodal network, outage probability is studied for a wide range of input power and optimal power values for both the modes are calculated.

\subsection{Future Work}

In this thesis, traffic optimization is considered only for voice and delay-sensitive data traffic. The rate allocation problem can be extended to other kinds of traffic like multimedia, video by considering their corresponding utility functions. The traffic can also be allocated to wired mode and wireless mode based on user decisions. By solving this high security traffic can be transmitted though wired channel. The power allocation problem is solved only for single-hop diamond relay multimodal network. This can be extended to multihop multimodal networks. Power allocation problem can also be extended for multimodal networks by considering interference from other wireless nodes. Multicasting information from source to other wireless nodes can be considered in multimodal cooperative networks. 


\section{References}

[1] M. Rumney, "IMT-Advanced: 4G Wireless Takes Shape in an Olympic Year," Agilent Measurement Journal, 2008.

[2] D.P. Bertsekas, R.G. Gallager, P. Humblet, and Massachusetts Institute of Technology. Center for Advanced Engineering Study, Data networks, Prentice-hall Englewood Cliffs, NJ, 1987.

[3] D. Ayyagari and A. Ephremides, "Admission control with priorities: approaces for multi-rate wireless system," Mobile Networks and Applications, vol. 4, no. 3, pp. 218, 1999.

[4] K. Zhang and K. Pahlavan, "An integrated voice/data system for mobile indoor radio networks," Vehicular Technology, IEEE Transactions on, vol. 39, no. 1, pp. 75-82, 2002.

[5] J.E. Wieselthier and A. Ephremides, "Fixed-and movable-boundary channel-access schemes for integrated voice/data wireless networks," Communications, IEEE Transactions on, vol. 43, no. 1, pp. 64-74, 2002.

[6] A. Sampath, S. Kumar, and J.M. Holtzman, "Power control and resource management for a multimedia CDMA wireless system," in Personal, Indoor and Mobile Radio Communications, 1995. PIMRC'95.'Wireless: Merging onto the Information Superhighway'., Sixth IEEE International Symposium on. IEEE, 2002, vol. 1, pp. 21-25.

[7] E. Calvo, J. Vidal, and J.R. Fonollosa, "Optimal resource allocation in relay-assisted cellular networks with partial CSI," Signal Processing, IEEE Transactions on, vol. 57, no. 7, pp. 2809-2823, 2009.

[8] C. Zhou, ML Honig, and S. Jordan, "Two-cell power allocation for wireless data based on pricing," in PROCEEDINGS OF THE ANNUAL ALLERTON CONFERENCE ON COMMUNICATION CONTROL AND COMPUTING. The University; 1998, 2001, vol. 39, pp. 1088-1097.

[9] C. Zhou, M.L. Honig, S. Jordan, and R. Berry, "Utility-based resource allocation for wireless networks with mixed voice and data services," in Computer Communications and Networks, 2002. Proceedings. Eleventh International Conference on. IEEE, 2002, pp. $485-488$. 
[10] C. Zhou, D. Qian, N. Pissinou, and K. Makki, "Resource allocation based on pricing for wireless multimedia networks," in Wireless Communications and Networking Conference, 2004. WCNC. 2004 IEEE. IEEE, 2004, vol. 1, pp. 477-482.

[11] F.P. Kelly, A.K. Maulloo, and D.K.H. Tan, "Rate control for communication networks: shadow prices, proportional fairness and stability," Journal of the Operational Research society, vol. 49, no. 3, pp. 237-252, 1998.

[12] J.W. Lee, M. Chiang, and A.R. Calderbank, "Price-based distributed algorithms for rate-reliability tradeoff in network utility maximization," Selected Areas in Communications, IEEE Journal on, vol. 24, no. 5, pp. 962-976, 2006.

[13] Y. Li, M. Chiang, A.R. Calderbank, and S.N. Diggavi, "Optimal rate-reliability-delay tradeoff in networks with composite links," Communications, IEEE Transactions on, vol. 57, no. 5, pp. 1390-1401, 2009.

[14] S. Stidham Jr, "Pricing and congestion management in a network with heterogeneous users," Automatic Control, IEEE Transactions on, vol. 49, no. 6, pp. 976-981, 2004.

[15] R.J. La and V. Anantharam, "Utility-based rate control in the Internet for elastic traffic," IEEE/ACM Transactions on Networking (TON), vol. 10, no. 2, pp. 272-286, 2002.

[16] X. Duan, Z. Niu, and J. Zheng, "Utility optimization and fairness guarantees for multimedia traffic in the downlink of DS-CDMA systems," in Global Telecommunications Conference, 2003. GLOBECOM'03. IEEE. IEEE, 2004, vol. 2, pp. 940-944.

[17] X. Duan, Z. Niu, and J. Zheng, "A dynamic utility-based radio resource management scheme for mobile multimedia DS-CDMA systems," in Global Telecommunications Conference, 2002. GLOBECOM'02. IEEE. IEEE, 2003, vol. 1, pp. 804-808.

[18] M.A. Visser and M. El Zarki, "Voice and data transmission over an 802.11 wireless network," in Personal, Indoor and Mobile Radio Communications, 1995. PIMRC'95.'Wireless: Merging onto the Information Superhighway'., Sixth IEEE International Symposium on. IEEE, 2002, vol. 2, pp. 648-652.

[19] W. Song, H. Jiang, W. Zhuang, and X. Shen, "Resource management for QoS support in cellular/WLAN interworking," Network, IEEE, vol. 19, no. 5, pp. 12-18, 2005.

[20] AE Gamal, "Capacity theorems for the relay channel," IEEE Transactions on Information Theory, vol. 25, no. 5, pp. 572-584, 1979.

[21] J.N. Laneman and G.W. Wornell, "Distributed space-time coded protocols for exploiting cooperative diversity in wireless networks," in Global Telecommunications Conference, 2002. GLOBECOM'02. IEEE. IEEE, 2003, vol. 1, pp. 77-81.

[22] J.N. Laneman, D.N.C. Tse, and G.W. Wornell, "Cooperative diversity in wireless networks: Efficient protocols and outage behavior," IEEE Transactions on Information theory, vol. 50, no. 12, pp. 3062-3080, 2004. 
[23] P. Gupta and P.R. Kumar, "The capacity of wireless networks," IEEE Transactions on information theory, vol. 46, no. 2, pp. 388-404, 2000.

[24] M. Franceschetti, M.D. Migliore, and P. Minero, "The capacity of wireless networks: information-theoretic and physical limits," Information Theory, IEEE Transactions on, vol. 55, no. 8, pp. 3413-3424, 2009.

[25] Ricky Hussmann and Daryl Reynolds, "Outage Probability for Multimodal Cooperative Networks," Accepted for IEEE International Conference on Communications, 2011.

[26] I. Rec, "G. 107-The E Model, a computational model for use in transmission planning," International Telecommunication Union, 1998.

[27] A. Goldsmith, Wireless communications, Cambridge Univ Pr, 2005.

[28] T.S. Rappaport, "Wireless communications: Principles and practice," 1996.

[29] Darly Reynolds, "Wireless communications," Course Notes, WVU, Spring 2009.

[30] V. Tarokh, H. Jafarkhani, and A.R. Calderbank, "Space-time block codes from orthogonal designs," IEEE Transactions on Information theory, vol. 45, no. 5, pp. 1456-1467, 1999.

[31] V. Tarokh, H. Jafarkhani, and AR Calderbank, "The application of orthogonal designs to wireless communication," in Information Theory Workshop, 1998. IEEE, 2002, pp. $46-47$.

[32] S.M. Alamouti, "A Simple Transmit Diversity Technique for Wireless Communications," communications, vol. 16, no. 8, pp. 1451-1458, 1998.

[33] C. Shannon, "E.(1948) A Mathematical Theory of Communication," Bell System Technical Journal, vol. 27, pp. 379-423.

[34] G. Song and Y. Li, "Utility-based resource allocation and scheduling in OFDM-based wireless broadband networks," Communications Magazine, IEEE, vol. 43, no. 12, pp. 127-134, 2005.

[35] E.C. Van Der Meulen, "Three-terminal communication channels," Advances in Applied Probability, vol. 3, no. 1, pp. 120-154, 1971.

[36] E.C. Van der Meulen, Transmission of information in a T-terminal discrete memoryless channel, University Microfilms, 1969.

[37] Krishna Jyothi Boppana and Daryl Reynolds, "Traffic Optimization for Multimodal Cooperative Networks," Accepted in Wireless @ Virginia Tech Symposium on Wireless Communications, 2011.

[38] S. Shenker, "Fundamental design issues for the future Internet," Selected Areas in Communications, IEEE Journal on, vol. 13, no. 7, pp. 1176-1188, 2002. 
[39] L. Kleinrock, Queueing Systems: Volume 2: Computer Applications, John Wiley \& Sons New York, 1976.

[40] S.P. Boyd and L. Vandenberghe, Convex optimization, Cambridge Univ Pr, 2004.

[41] Dr. Daryl Reynolds, "Communications research at wvu," seminar, WVU, Fall 2009. 


\section{A Proof for Maximizing Total Utility in Data and Voice Networks}

The Lagrange equation for solving rate optimization problem after substituting utility functions of data (3.3) and voice (3.8) is given by

$$
\begin{aligned}
\Lambda\left(R_{d}, R_{v}, \lambda\right)= & U_{d}\left(R_{d}, \rho_{d}, \delta_{d}\right)+U_{v}\left(\rho_{v}, R_{v}\right)+\lambda\left(R_{d}+R_{v}-R\right) \\
= & w_{d} \frac{r_{d} \rho_{d}-\left(r_{d} \rho_{d}\right)^{\text {min }}}{\left(r_{d} \rho_{d}\right)^{\text {max }}-\left(r_{d} \rho_{d}\right)^{\text {min }}}-\left(1-w_{d}\right) \frac{\frac{K}{2}\left(\frac{1}{R}+\frac{1}{R-r_{d}}\right)-\delta_{s}^{\text {min }}}{\delta_{d}^{\text {max }}-\delta_{s}^{\text {min }}} \\
& +R_{a}-\alpha_{1} \frac{K}{2}\left(\frac{1}{R}+\frac{1}{R-R_{v}}\right)-\alpha_{2}\left(\frac{K}{2}\left(\frac{1}{R}+\frac{1}{R-R_{v}}\right)-\alpha_{3}\right) H \\
& -\beta_{1}-\beta_{2} \log \left(1+100 \beta_{3}\left(1-\rho_{v}\right)\right)+\lambda\left(R_{d}+R_{v}-R\right) .
\end{aligned}
$$

Let $a=\left(R_{d} \rho_{d}\right)^{\max }-\left(R_{d} \rho_{d}\right)_{\min }$ and $b=\left(\delta_{d}^{\max }-\delta_{d}^{\min }\right)$. Substituting the values of $a$ and $b$ in $(\mathrm{A}-19)$ we get

$$
\begin{aligned}
\Lambda\left(R_{d}, R_{v}, \lambda\right)= & w_{d} \frac{R_{d} \rho_{d}-\left(r_{d} \rho_{d}\right)^{\text {min }}}{a}-\left(1-w_{d}\right) \frac{\frac{K}{2}\left(\frac{1}{R}+\frac{1}{R-r_{d}}\right)-\delta_{s}^{\text {min }}}{b} \\
& +R_{a}-\alpha_{1} \frac{K}{2}\left(\frac{1}{R}+\frac{1}{R-R_{v}}\right)-\alpha_{2}\left(\frac{K}{2}\left(\frac{1}{R}+\frac{1}{R-R_{v}}\right)-\alpha_{3}\right) H \\
& -\beta_{1}-\beta_{2} \log \left(1+100 \beta_{3}\left(1-\rho_{v}\right)\right)+\lambda\left(R_{d}+R_{v}-R\right) .
\end{aligned}
$$

Differentiating the equation (A-1) w.r.t $R_{d}$ we get

$$
\frac{\partial \Lambda}{\partial R_{d}}=\frac{w_{d}\left(\rho_{d}\right)}{a}-\frac{\left(1-w_{d}\right) K}{2 b}\left[\frac{1}{\left(R-R_{d}\right)^{2}}\right]+\lambda
$$

Differentiating the equation (A-2) w.r.t $R_{v}$ we get

$$
\frac{\partial \Lambda}{\partial R_{v}}=-\frac{K}{2} \alpha_{1}\left[\frac{1}{\left(R-R_{v}\right)^{2}}\right]-\frac{H \alpha_{1} K}{2}\left[\frac{1}{\left(R-R_{v}\right)^{2}}\right]+\lambda .
$$

Using KKT conditions we can equate equations (A-3) and (A-4) to zero. Applying KKT condition to equation (A-4), we will get the value of $\lambda$ as

$$
\lambda=\frac{K}{2} \alpha_{1} \frac{1}{R_{d}^{2}}+\frac{\alpha_{2} H K}{2} \times \frac{1}{R_{d}^{2}}
$$


Substituting the value of $\lambda$ from (A-5) and $R_{v}=R-R_{d}$ in (A-3) and equating to zero we get

$$
\frac{w_{d}\left[\rho_{d}\right]}{a}-\frac{1-w_{d}}{b} \times \frac{K}{2}\left[\frac{a}{\left(R-R_{d}\right)^{2}}\right]+\frac{K \alpha_{1}}{2} \times \frac{1}{R_{d}^{2}}+\frac{\alpha_{2} H K}{2 R_{d}^{2}}=0 .
$$

The equation (A-6) is a quartic function in $R_{d}$. Solving the equation numerically for the range of values from 0 to $R$ in matlab gives the optimal value of $R_{d}$ and $R_{v}=R-R_{d}$ gives the optimal value of $R_{v}$.

\section{B Proof for Rate Maximization in a Source-Destination Pair Multimodal Network}

The Lagrange equation for the capacity optimization problem is formulated as

$$
\Lambda\left(P_{w}, P_{w l}, \lambda\right)=\frac{B_{w}}{B_{w l}} \log _{2}\left(1+\left|h_{w}\right|^{2} \frac{P_{w}}{N_{w} B_{w}}\right)+\log _{2}\left(1+\left|h_{w l}\right|^{2} \frac{P_{w l}}{N_{w l} B_{w l}}\right)+\lambda\left(P_{w}+P_{w l}-P\right) .
$$

Differentiating equation (B-7) partially w.r.t $P_{w}$

$$
\frac{\partial \Lambda}{\partial P_{w}}=B_{w}\left(\frac{1}{1+\frac{\left|h_{w}\right|^{2} P_{w}}{N_{w} B_{w}}}\right)\left(\frac{h_{w}}{N_{w} B_{w}}\right)+\lambda
$$

Differentiating equation (B-7) partially w.r.t $P_{w l}$

$$
\frac{\partial \Lambda}{\partial P_{w}}=B_{w l}\left(\frac{1}{1+\frac{\left|h_{w l}\right|^{2} P_{w l}}{N_{w l} B_{w l}}}\right)\left(\frac{\left|h_{w l}\right|^{2}}{N_{w l} B_{w l}}\right)+\lambda
$$

Differentiating equation (B-7) partially w.r.t $\lambda$ and equating to zero

$$
\frac{\partial \Lambda}{\partial \lambda}=P_{w}+P_{w l}-P=0
$$

Using KKT conditions we can equate (B-8) and (B-9) to zero. Equating (B-7) to zero we will get the value of $\lambda$ as

$$
\lambda=B_{w l}\left(\frac{1}{1+\frac{\left|h_{w l}\right|^{2} P_{w l}}{N_{w l} B_{w l}}}\right)\left(\frac{\left|h_{w l}\right|^{2}}{N_{w l} B_{w l}}\right) .
$$

Substituting the value of $\lambda$ in (B-9)

$$
\left(B_{w}\left|h_{w}\right|^{2}\right)\left(N_{w l} B_{w l}+\left|h_{w l}\right|^{2}\left(P-P_{w}\right)\right)-\left(\left|h_{w l}\right|^{2} B_{w l}\right)\left(N_{w} B_{w}+\left|h_{w}\right|^{2} P_{w}\right)=0
$$


Value of $P_{w}$ form the equation (B-12) is,

$$
P_{w}=\frac{N_{w l} B_{w} B_{w l}\left|h_{w}\right|^{2}+B_{w}\left|h_{w}\right|^{2}\left|h_{w l}\right|^{2} P-\left|h_{w l}\right|^{2} B_{w l} B_{w} N_{w}}{\left|h_{w}\right|^{2}\left|h_{w l}\right|^{2}\left(B_{w}+B_{w l}\right)} .
$$

So, wireless power $P_{w l}$ is

$$
\begin{aligned}
P_{w l} & =P-P_{w} \\
& =P-\left(\frac{N_{w l} B_{w} B_{w l}\left|h_{w}\right|^{2}+B_{w}\left|h_{w}\right|^{2}\left|h_{w l}\right|^{2} P-\left|h_{w l}\right|^{2} B_{w l} N_{w} B_{w}}{\left|h_{w}\right|^{2}\left|h_{w l}\right|^{2}\left(B_{w}+B_{w l}\right)}\right) .
\end{aligned}
$$

\section{Proof for Outage Minimization in a Source-Destination Pair Multimodal Network}

The Lagrange equation for this problem from equation (4.12) is given by

$$
\Lambda_{1}\left(P_{w}, P_{w l}, \lambda_{1}\right)=\frac{2^{R-\left(B_{w} / B_{w l}\right) \log \left(1+\frac{\left|h_{w}\right|^{2} P_{w}}{N_{w} B_{w}}\right)}-1}{\frac{P_{w l}}{N_{w l} B_{w l}}}+\lambda_{1}\left(P_{w}+P_{w l}-P\right) .
$$

Differentiating the equation $(\mathrm{C}-16)$ with respect to $P_{w}$ and equating to zero we get

$$
\frac{\partial \Lambda_{1}}{\partial P_{w}}=\left(2^{R-\left(B_{w} / B_{w l}\right) \log \left(1+\frac{\left|h_{w}\right|^{2} P_{w}}{N_{w} B_{w}}\right)}\right)\left(\frac{N_{w l}}{P_{w l}}\right) \frac{-\left|h_{w}\right|^{2} B_{w}}{\left(N_{w} B_{w}+\left|h_{w}\right|^{2} P_{w}\right)}+\lambda_{1}=0 .
$$

Differentiating the equation $(\mathrm{C}-16)$ with respect to $P_{w l}$ and equating to zero we get

$$
\frac{\partial \Lambda_{1}}{\partial P_{w l}}=\left(2^{R-\left(B_{w} / B_{w l}\right) \log \left(1+\frac{\left|h_{w}\right|^{2} P_{w}}{B_{w} N_{w}}\right)}-1\right)\left(N_{w l} B_{w l} \frac{-1}{P_{w l}^{2}}\right)+\lambda_{1}=0 .
$$

Differentiating the equation (C-16) with respect to $\lambda_{1}$ and equating to zero

$$
\frac{\partial \Lambda_{1}}{\partial \lambda_{1}}=P_{w}+P_{w l}-P=0
$$

Equations (C-17) and (C-18) are two simultaneous equations with two unknown variables $P_{w}$ and $P_{w l}$. These two equations are solved in matlab numerically to get the optimal values of $P_{w}$ and $P_{w l}$. 


\section{Proofs for Minimizing Outage probability for Coop- erative Diamond Relay Multimodal Network}

\section{D.1 Wire between Source and Relay}

The expression for information outage probability for the network with wire between source and relay is [25]

$$
\begin{aligned}
P_{r}[I<R] \approx & \left(\frac{2^{2 R}-1}{2 \mathrm{SNR} / m}\right) \times \lambda_{s, d(s)}\left[\left(\frac{1}{1-P_{w}}\right)^{2} \times \lambda_{s, r_{w}}\left(2^{2 R-\frac{B w}{B_{w l}} \times \log \left(1+\frac{2 P_{w}}{m B_{w} / B_{w l}} \mathrm{SNR}|h|^{2}\right)}\right) \times \lambda_{s, r_{1}}\right. \\
& +\left(\frac{1}{1-P_{w}}\right) \times \lambda_{s, r_{w}} \times\left(2^{2 R-\frac{B w}{B_{w l}} \log \left(1+\frac{2 P_{w}}{m B_{w} / B_{w l}} \mathrm{SNR}|h|^{2}\right)}\right) \times \lambda_{r_{1}, d(s)} \times A_{1}\left(2^{2 R}-1\right) \\
& +\left(\frac{1}{1-P_{w}}\right) \times \lambda_{r_{w}, d(s)} \times \lambda_{s, r_{1}} \times A_{1}\left(2^{2 R}-1\right)+\lambda_{r_{w}, d(s)} \\
& \left.\times \lambda_{r_{1}, d(s)} \times A_{2}\left(2^{2 R}-1\right)\right] .
\end{aligned}
$$

The partial derivative of (D-20) w.r.t to $P_{w}$ is

$$
\begin{aligned}
\frac{\partial P_{r}}{\partial P_{w}}= & \frac{2^{2 R}-1}{\frac{2 \mathrm{SNR}}{m} \times \lambda_{s, d(s)}}\left\{\left[\lambda_{s, r_{w}} \times\left(2^{2 R-\frac{B_{w}}{B_{w l}} \log \left(1+\frac{2 P_{w}}{m \frac{B w}{B_{w l}}} \mathrm{SNR}|h|^{2}\right.}\right)\right)\right. \\
& \left.\times\left(\frac{2 \lambda_{s, r_{1}}}{\left(1-P_{w}\right)^{3}}+\frac{1}{\left(1-P_{w}\right)^{2}} \lambda_{r_{1}, d(s)} A_{1}\left(2^{2 R}-1\right)\right)\right]+\left[\left(\frac{\lambda_{s, r_{1}}}{\left(1-P_{w}\right)^{2}}+\frac{1}{\left(1-P_{w}\right)} \lambda_{r_{1}, d(s)}\right.\right. \\
& \left.\left.A_{1}\left(2^{2 R}-1\right)\right) \times\left(\lambda_{s, r_{w}} \times\left(2^{2 R-\frac{B_{w}}{B_{w l}} \log \left(1+\frac{2 P_{w}}{m \frac{B w}{B_{w l}}} \mathrm{SNR}|h|^{2}\right.}\right)\right)\left(\frac{\frac{-2}{m} \mathrm{SNR}|h|^{2}}{1+\frac{2 P_{w}}{m \frac{B w}{B_{w l}}} \mathrm{SNR}|h|^{2}}\right)\right] \\
& \left.+\frac{1}{\left(1-P_{w}\right)^{2}} \lambda_{r_{w} d(s)} \lambda_{s, r_{1}} A_{1}\left(2^{2 R}-1\right)\right\} .
\end{aligned}
$$

The optimal $P_{w}$ value in the equation (D-20) is found using the numerical values of (D-21) in matlab. 


\section{D.2 Wire between Relay and Destination}

The outage probability expression for the network with wire between relay and destination is $[25]$

$$
\begin{aligned}
P_{r}(I<R)= & \frac{1}{2 \mathrm{SNR} / m} \times \lambda_{r_{w}, d(s)} \times\left[\frac { \lambda _ { r _ { w } , d ( s ) } } { 1 - P _ { w } } \left(\left(2^{2 \tilde{R}}-1\right)^{2} \times\left(2^{2 R}-1\right) \times \lambda_{s, r_{2}} \times\right.\right. \\
& \left.A_{1}\left(2^{2 \tilde{R}}-1\right)+\left(2^{2 \tilde{R}}-1\right)^{3} \times \lambda_{r_{2}, d(s)} \times A_{2}\left(2^{2 \tilde{R}}-1\right)\right)+\left(\left(2^{2 R}-1\right)^{3} \times \lambda_{s, r_{w}}\right. \\
& \left.\left.\times\left(\lambda_{s, r_{2}}+\lambda_{r_{2}, d(s)} \times A_{1}\left(2^{R}-1\right)\right)\right)\right] .
\end{aligned}
$$

Let $t=2^{2 R}-1, t_{1}=2^{2 \tilde{R}}-1, f=A_{1}\left(2^{2 R}-1\right), f_{1}=A_{1}\left(2^{\tilde{R}}-1\right), f_{2}=A_{2}\left(2^{2 \tilde{R}}-1\right)$. Then the equation (D-22) can be written as

$$
\begin{aligned}
P_{r}(I<R)= & \frac{1}{2 \mathrm{SNR} / m} \times \lambda_{r_{w}, d(s)} \times\left[\frac { \lambda _ { r _ { w } , d ( s ) } } { 1 - P _ { w } } \left(t_{1}^{2} \times t \times \lambda_{s, r_{2}} \times f_{1}\right.\right. \\
& \left.\left.+t_{1}^{3} \times \lambda_{r_{2}, d(s)} \times f_{2}\right)+\left(t^{3} \times \lambda_{s, r_{w}} \times\left(\lambda_{s, r_{2}}+\lambda_{r_{2}, d(s)} \times f_{1}\right)\right)\right]
\end{aligned}
$$

Differentiating the equation (D-23), we get

$$
\begin{aligned}
\frac{\partial P_{r}}{\partial P_{w}}= & \frac{\lambda_{r_{w}, d(s)}}{1-P_{w}}\left[2 t_{1} \times \frac{\mathrm{d} t_{1}}{\mathrm{~d} P_{w}} \times t \times \lambda_{s, r_{2}} \times f_{1}+t_{1}^{2} \times t \times \lambda_{s, r_{2}} \times \frac{\mathrm{d} f_{1}}{\mathrm{~d} P_{w}}+t_{1}^{2} \times t \times \lambda_{s, r_{2}} \times f_{1}\right. \\
& \left.+t_{1}^{3} \times \lambda_{r_{2}, d(s)} \times \frac{\mathrm{d} f_{2}}{\mathrm{~d} P_{w}}+3 t_{1}^{2} \times \frac{\mathrm{d} t_{1}}{\mathrm{~d} P_{w}} \times \lambda_{r_{2}, d(s)} \times f_{2}\right]
\end{aligned}
$$

Numerical values of (D-24) are calculated to get the optimal value of $P_{w}$ for the equation (D-22) in matlab. 